\title{
Consistent tangent matrices for density-dependent finite plasticity models
}

\author{
Agustí Pérez-Foguet, Antonio Rodríguez-Ferran and Antonio Huerta*, ${ }^{\dagger}$ \\ Departament de Matemàtica Aplicada III, E.T.S. de Ingenieros de Caminos, Canales y Puertos, \\ Universitat Politècnica de Catalunya, Jordi Girona 1, E-08034 Barcelona, Spain
}

\begin{abstract}
SUMMARY
The consistent tangent matrix for density-dependent plastic models within the theory of isotropic multiplicative hyperelastoplasticity is presented here. Plastic equations expressed as general functions of the Kirchhoff stresses and density are considered. They include the Cauchy-based plastic models as a particular case. The standard exponential return-mapping algorithm is applied, with the density playing the role of a fixed parameter during the nonlinear plastic corrector problem. The consistent tangent matrix has the same structure as in the usual density-independent plastic models. A simple additional term takes into account the influence of the density on the plastic corrector problem. Quadratic convergence results are shown for several representative examples involving geomaterial and powder constitutive models.
\end{abstract}

KEY WORDS: finite strain multiplicative plasticity; Cauchy stresses; consistent tangent operators; geomaterial plastic models; powder compaction; arbitrary Lagrangian-Eulerian (ALE) formulation

\section{INTRODUCTION}

Consistent tangent matrices [1,2] are an essential ingredient for the efficient solution via implicit methods of complex problems in non-linear computational mechanics. Consistent tangent matrices are needed to solve the elastoplastic boundary value problem with quadratic convergence, via a full Newton-Raphson linearization. They are computed from the consistent tangent moduli at Gauss-point level.

The expression of the consistent tangent moduli for a wide variety of material models can be found in the literature. Consistent tangent moduli for different small-strain elastoplastic models and time-integration rules can be found in References [1-5] among many others.

\footnotetext{
* Correspondence to: Antonio Huerta, Departament de Matemàtica Aplicada III, E.T.S. de Ingenieros de Caminos, Canales y Puertos, Universitat Politècnica de Catalunya, Jordi Girona 1, E-08034 Barcelona, Spain

† E-mail: antonio.huerta@upc.es

Contract grant sponsor: Ministerio de Educación y Cultura; contract/grant number: TAP98-0421

Contract grant sponsor: Comisión Interministerial de Ciencia y Technología; contract/grant number: 2FD97-1206
} 
In the context of finite-strain modelling of isotropic materials, both multiplicative hyperelastoplasticity theory with logarithmic strain measures and time-integration based on the exponential mapping are a standard approach [6-9]. From a computational point of view, its attractiveness stems from the fact that it leads to the same return-mapping algorithm of the infinitesimal theory, and, therefore, the same linearization of the plastic corrector nonlinear equations. This approach was developed initially for plastic incompressible materials in terms of the Kirchhoff stresses $[6,9]$. Extensions to include thermal coupling [10], damage effects [11,12], and specific geomaterial plastic models [13-16] have been presented. Applications to consolidation problems have also been developed [17-19]. In all these cases, the consistent linearization of the numerical time-integration algorithm is included.

A key issue in the application of the multiplicative hyperelastoplasticity theory to geomaterials is the choice of the stress measure for the constitutive equations. This affects directly how to model the influence of the density, or, equivalently, the volumetric deformation, on the plastic behaviour. Recently, Meschke and Liu [20] have presented a re-formulation of the returnmapping algorithm in terms of the Cauchy stresses. They present a discussion on the role of the stress measure that needs to be chosen as the argument of the plastic model and comparative examples. The corresponding consistent tangent moduli are also included.

A more general way to model the influence of the volumetric deformation on the plastic behaviour is to consider density-dependent plastic equations, this is, yield function and flow rules expressed in terms of the density and the Kirchhoff stresses. Here, it is shown that the standard exponential return-mapping algorithm applies to this type of models. Moreover, the

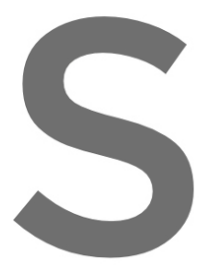

consistent tangent

Reference $[20]$ are,

models.

On the other hand, in powder compaction s tion of the stresses and the density are us
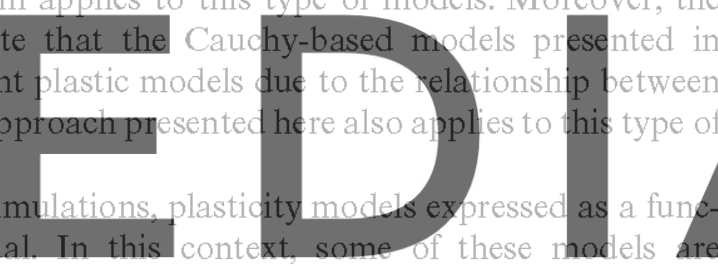

formulated within the multiplicative hyperelastoplasticity theory, with the Kirchhoff stresses as

return-mapping algorithms are devised, and, in some cases the corresponding consistent tangent

moduit are presented $[21,22,24]$. The approach presented here, without simplifications of the general kinematic framework, has been applied successfully to powder compaction modelling in Reference [25]. In this reference it is shown that considering the influence of large elastic deformations in this type of problems does not represent any drawback from a modelling point of view.

An outline of the paper follows. The problem is stated in Section 2. The constitutive model and the standard numerical time-integration algorithm are briefly reviewed. The consistent linearization of the algorithm is presented in Section 3. First, the expression for density-independent models is shown. After that, the extension to the density-dependent case is devised. In Section 4, representative examples are discussed in detail and the convergence results are highlighted. Three elastoplastic models with different degree of computational difficulty are used: a Drucker-Prager model [20], an elliptic model [21,22,26,27] and a cone-cap model [23,24,28-31]. The arbitrary Lagrangian-Eulerian formulation for multiplicative elastoplasticity presented in Reference [32] is used in some of the examples to avoid mesh distortion. The main conclusions are summarized in Section 5. 


\section{CONSISTENT TANGENT MATRICES}

\section{PROBLEM STATEMENT}

This section presents a brief description of the problem. First, the constitutive equations are summarized. After that, key issues of the integration scheme are described. See Simo [6,9], Meschke and Liu [20] and references therein for further details on the general framework.

Here, the dependence of the plastic model on the density is included in the yield function and the flow rule in a general way. Moreover, it is shown that the density has no influence on the standard exponential return-mapping algorithm.

\subsection{Constitutive model}

Let $\Omega \subset \mathbb{R}^{n_{\mathrm{dim}}}\left(n_{\mathrm{dim}}=2,3\right)$ be the material configuration of a continuum body with particles labelled by $X \in \Omega$. The motion of $\Omega$ is described by the one-parameter family of mappings $\varphi_{t}: \Omega \mapsto \mathbb{R}^{n_{\mathrm{dim}}}$ with $t \in[0, T]$ the time-like parameter. Let $\Omega_{t}=\varphi_{t}(\Omega)$ be the placement of the body at time $t$ and $x=\varphi_{t}(X)=\varphi(X, t) \in \Omega_{t}$ the position of the material particle $X$. In that context, the deformation gradient,

$$
\boldsymbol{F}(\boldsymbol{X}, t)=\frac{\partial \varphi}{\partial \boldsymbol{X}}(\boldsymbol{X}, t)
$$

is assumed to be locally decomposed into elastic and plastic parts as
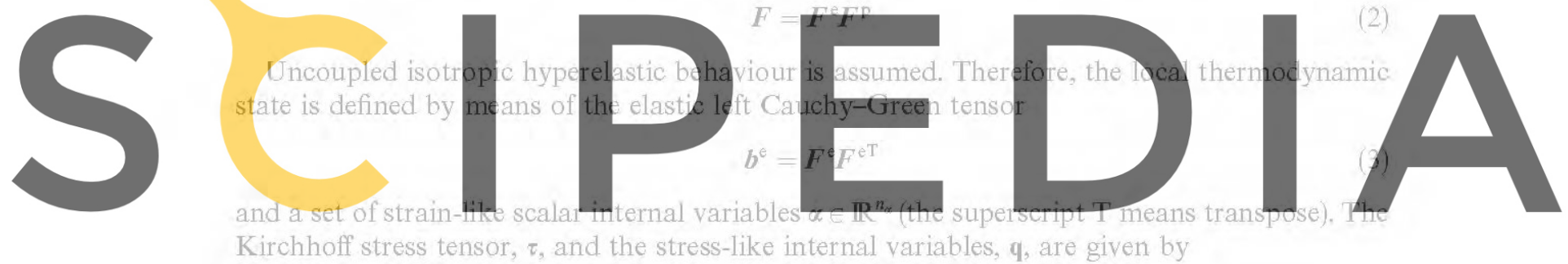

Kirchhoff stress tensor, $\tau$, and the stress-like internal variables, $q$, are given by

Register for free at https//www.scipedia.com to download the version without the watermark

$$
\begin{aligned}
& \tau=2 \frac{\mathrm{d}}{\mathrm{d} b^{\mathrm{e}}} b^{\mathrm{e}} \\
& \mathbf{q}=-\frac{\mathrm{d} \boldsymbol{W}^{\mathrm{p}}}{\mathrm{d} \boldsymbol{\alpha}}
\end{aligned}
$$

where $W^{\mathrm{e}}$ and $W^{\mathrm{p}}$ are the elastic and plastic parts of the free-energy function per unit of undeformed volume [33]. The Cauchy stress tensor is given by

$$
\sigma=\frac{\tau}{\operatorname{det}(\boldsymbol{F})}
$$

The mass conservation equation reads, in material formulation,

$$
\rho(\boldsymbol{X}, t)=\frac{\rho_{0}(\boldsymbol{X})}{\operatorname{det}(\boldsymbol{F})}
$$

where $\rho(\boldsymbol{X}, t)$ and $\rho_{0}(\boldsymbol{X})$ are the densities of the particle $\boldsymbol{X}$ at times $t$ and $t=0$, respectively. In powder compaction modelling, Equation (6) is rewritten in dimensionless format, in terms of the 
relative density, $\eta(\boldsymbol{X}, t)[21-23,30]$. Both sides of Equation (6) are divided by the solid density of the compacted material, which is taken as a reference value. This leads to the equivalent expression

$$
\eta(\boldsymbol{X}, t)=\frac{\eta_{0}(\boldsymbol{X})}{\operatorname{det}(\boldsymbol{F})} .
$$

The plastic response of the material is assumed isotropic and density-dependent. The dependence on the density is incorporated through $\eta$. A generic yield function

$$
f(\tau, \mathbf{q}, \eta)=0
$$

and flow rules

$$
\begin{gathered}
\mathscr{L}_{v} \boldsymbol{b}^{\mathrm{e}}=-2 j \mathbf{m}_{\tau}(\tau, \mathbf{q}, \eta) \boldsymbol{b}^{\mathrm{e}} \\
\dot{\alpha}=j \mathbf{m}_{q}(\tau, \mathbf{q}, \eta)
\end{gathered}
$$

are considered, with $\mathscr{L}_{v}$ the Lie derivative with respect to the spatial velocity, $\boldsymbol{v}, \mathbf{m}_{\mathrm{v}}$ and $\mathbf{m}_{q}$ the corresponding flow directions, and $\dot{\gamma}$ the plastic multiplier. The plastic multiplier is determined with the classical Kuhn-Tucker conditions
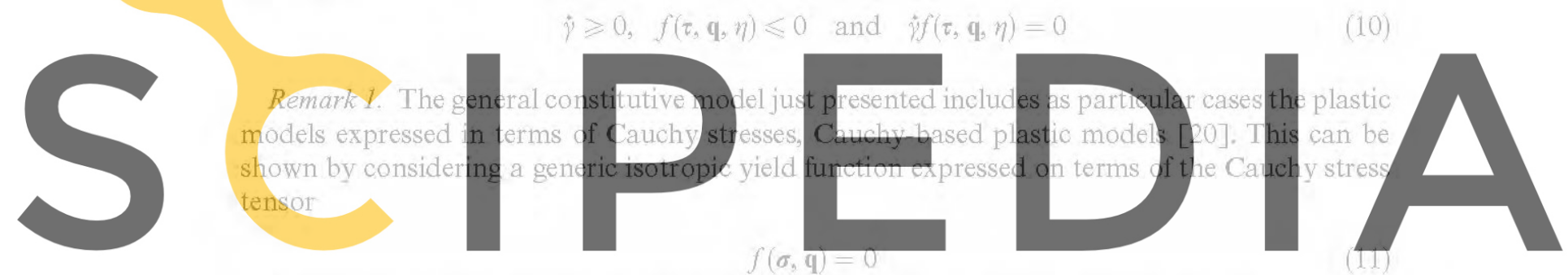

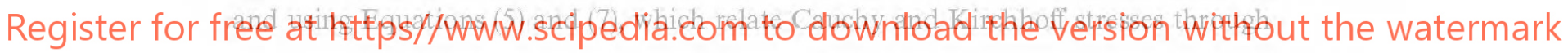

$$
\sigma=\frac{\eta}{\eta_{0}} \tau
$$

Then, a density-dependent yield function expressed in terms of the Kirchhoff stress tensor, $\tilde{f}\left(\tau, \boldsymbol{q}, \eta / \eta_{0}\right)$, can be defined simply by substituting Equation (12) into (11):

$$
f(\boldsymbol{\sigma}, \mathbf{q})=\tilde{f}\left(\tau, \mathbf{q}, \eta / \eta_{0}\right)=0
$$

The function $\tilde{f}$ is not unique. Thus, the most convenient expression from a computational point of view should be chosen. On the other hand, $\tilde{f}$ depends on $\eta$ only through $\eta / \eta_{0}$. So an arbitrary scale can be chosen, for instance $\eta_{0}=1$ for all particles $\boldsymbol{X}$. See the example presented in Section 4 and the appendix for further details.

The other components of the Cauchy-based models, i.e. hyperelastic relationships and flow rules, are equal to those presented for density-dependent Kirchhoff-based models, see equation (9). This can be shown following the developments of Meschke and Liu [20] with a yield function $\tilde{f}$ given by Equation (13). Therefore, the numerical time-integration algorithm and consistent tangent moduli presented in the following apply to both types of models. 
Remark 2. The relative density affects the plastic constitutive equations, but not in the hyperelastic relationship. Therefore, following standard arguments [6], the local rate of dissipation per unit of reference volume, $\mathscr{D}$, can be expressed as

$$
\mathscr{D}=\dot{\gamma}\left(\tau: \mathbf{m}_{\tau}(\tau, \mathbf{q}, \eta)+\mathbf{q}^{\mathrm{T}} \mathbf{m}_{\mathbf{q}}(\tau, \mathbf{q}, \eta)\right) \geqslant 0
$$

where ':' means double contraction.

The maximum plastic dissipation principle leads to the associative flow rules

$$
\mathbf{m}_{\tau}(\tau, \mathbf{q}, \eta)=\frac{\partial f}{\partial \tau}(\tau, \mathbf{q}, \eta) \quad \text { and } \quad \mathbf{m}_{\mathbf{q}}(\tau, \mathbf{q}, \eta)=\frac{\partial f}{\partial \mathbf{q}}(\tau, \mathbf{q}, \eta)
$$

where $f(\tau, \mathbf{q}, \eta)$ is assumed to be convex on $\tau$ and $\mathbf{q}$.

\subsection{Numerical time-integration}

The evolution from time ${ }^{n} t$ to time ${ }^{n+1} t={ }^{n} t+\Delta t$ of the different magnitudes associated with a prescribed material particle $X$ is computed by means of the time-integration of the state variables $\boldsymbol{b}^{\mathrm{e}}, \boldsymbol{\alpha}$ and $\eta$.

Let us assume that ${ }^{n} \boldsymbol{x}=\boldsymbol{x}\left(\boldsymbol{X},{ }^{n} t\right),{ }^{n} \boldsymbol{b}^{\mathrm{e}},{ }^{n} \boldsymbol{\alpha}$ and ${ }^{n} \eta$ are known values referred to time ${ }^{n} t$ and that the incremental displacement
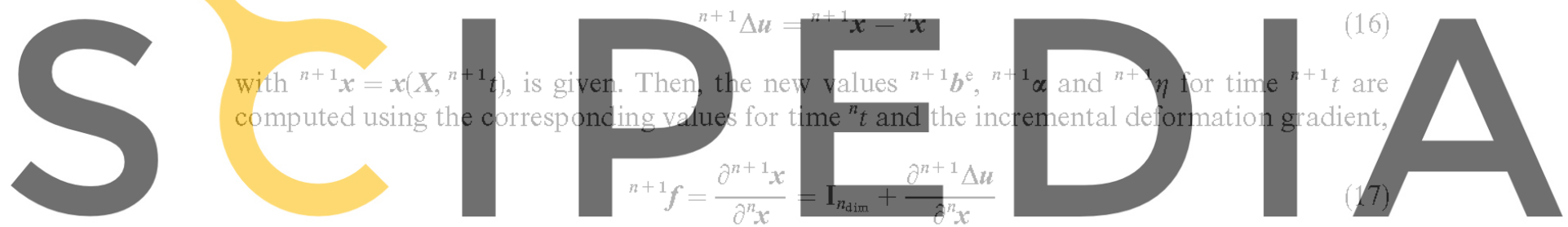

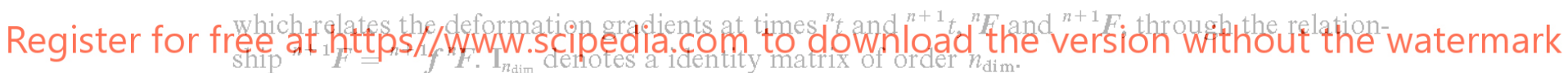

The relative density, $\eta$, is integrated exactly (in the sense that no numerical time-integration

scheme is used) because of the Lagrangian expression of the mass conservation principle, Equation (7), which leads to

$$
{ }^{n+1} \eta=\frac{\eta_{0}}{\operatorname{det}\left({ }^{n+1} \boldsymbol{F}\right)}=\frac{{ }^{n} \eta}{\operatorname{det}\left({ }^{n+1} \boldsymbol{f}\right)}
$$

The values of ${ }^{n+1} b^{e}$ and ${ }^{n+1} \boldsymbol{\alpha}$ are obtained by means of the standard elastic predictor-plastic corrector split strategy applied to Equations (8)-(10). Remarkably, the dependence of the constitutive equations on the density does not modify the algorithm. The value of ${ }^{n+1} \eta$ is given by Equation (18), and therefore it plays the role of a fixed parameter.

The result of the elastic predictor step is the so-called trial state. It is defined by

$$
\boldsymbol{b}_{\mathrm{tr}}^{\mathrm{e}}={ }^{n+1} \boldsymbol{f}^{n} \boldsymbol{b}^{\mathrm{e} n+1} \boldsymbol{f}^{\mathrm{T}} \text { and } \quad \boldsymbol{\alpha}_{\mathrm{tr}}={ }^{n} \boldsymbol{\alpha}
$$

If the trial state is admissible, $f\left(\tau_{\mathrm{tr}}, \mathbf{q}_{\mathrm{tr}},{ }^{n+1} \eta\right) \leqslant 0$, the state at time ${ }^{n+1} t$ is set equal to the trial state. If it is not, a plastic corrector step is computed. 
The plastic correction step requires the approximation of the flow rules, Equations (9a) and (9b). A standard approximation consists in the use of the exponential map and the backward Euler integration scheme $[6,7,9]$. Under the previous isotropy assumptions, this approach leads to a non-linear system of equations with the same structure as that of infinitesimal elastoplasticity.

In order to obtain the non-linear system of equations, three vectors of $\mathbb{R}^{n_{\mathrm{dim}}}$ are defined: $\varepsilon_{\mathrm{tr}}^{\mathrm{e}}$ ${ }^{n+1} \varepsilon^{e}$ and ${ }^{n+1} \bar{\tau}$. The components of $\varepsilon_{\mathrm{tr}}^{\mathrm{e}}$ and ${ }^{n+1} \varepsilon^{\mathrm{e}}$ are proportional to the eigenvalues of the tensors $\boldsymbol{b}_{\mathrm{tr}}^{\mathrm{e}}$ and ${ }^{n+1} \boldsymbol{b}^{\mathrm{e}}$, respectively, and the components of ${ }^{n+1} \bar{\tau}$ are the eigenvalues of ${ }^{n+1} \tau$ :

$$
\begin{aligned}
& \boldsymbol{b}_{\mathrm{tr}}^{\mathrm{e}}=\sum_{i=1}^{n_{\mathrm{dim}}}\left(\exp \left(\left[\varepsilon_{\mathrm{tr}}^{\mathrm{e}}\right]_{i}\right)\right)^{2} \mathbf{n}_{\mathrm{tr}}^{i} \otimes \mathbf{n}_{t r}^{i} \\
& n^{+1} b^{\varepsilon}=\sum_{i=1}^{n_{\text {dim }}}\left(\exp \left(\left[\left[^{n+1} \varepsilon_{\varepsilon}^{\varepsilon}\right]_{i}\right)\right)^{2} \mathbf{n}_{\mathrm{tr}}^{i} \otimes \mathbf{n}_{\mathrm{tt}}^{i}\right. \\
& { }^{n+1} \tau=\sum_{i=1}^{n+\operatorname{dim}}\left[{ }^{n+1} \bar{\tau}\right]_{i} \mathbf{n}_{\mathrm{rr}}^{i} \otimes \mathbf{n}_{\mathrm{tr}}^{i}
\end{aligned}
$$

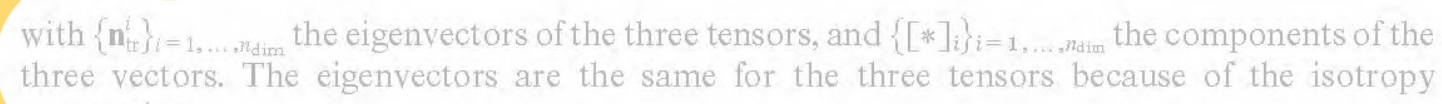
assumptions.
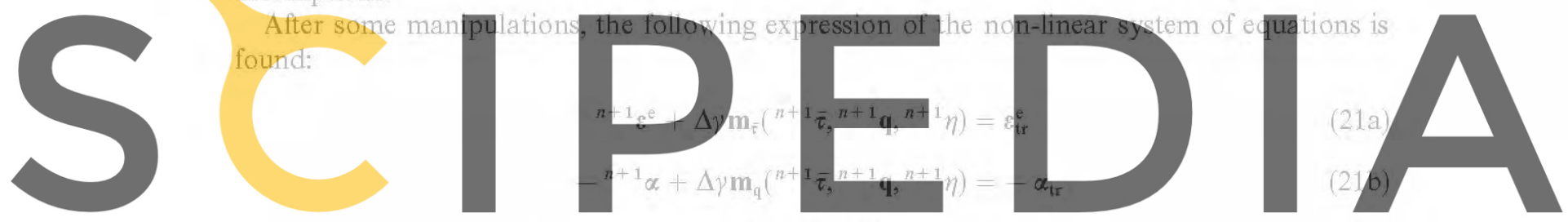

$f\left({ }^{n+1} \bar{\tau}_{,}{ }^{n+1} q_{0}{ }^{n+1} \eta\right)=0$

(21c)

Register for free at https//www. scipedia.com to download the version without the watermark

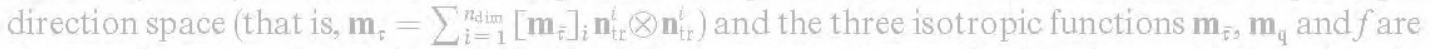
expressed as functions of $\bar{\imath}$. Equations (21) are complemented with the iestriction $\Delta \gamma \geqslant 0$, Equation (4b) and

$$
\bar{\tau}=\frac{\mathrm{d} \bar{W}^{e}}{\mathrm{~d} \varepsilon^{e}}
$$

with $\bar{W}^{\mathrm{e}}\left(\varepsilon^{\mathrm{e}}\right)$ defined so that Equation (22) is equivalent to the hyperelastic relationship (4a). Once Equations (21a)-(21c) are solved, the variables at time ${ }^{n+1} t$ are fully determined.

\section{CONSISTENT TANGENT MODULI FOR DENSITY-DEPENDENT FINITE PLASTICITY MODELS}

The linearization of the previous algorithm with respect to the gradient of the incremental displacement is needed to solve the discrete boundary value problem with quadratic convergence. 
In the following, the expression of the consistent tangent moduli for density-independent plastic models is briefly reviewed. After that, the general expression including the density influence is derived.

\subsection{Expression for density-independent models}

The linearized problem is completely specified once the following consistent tangent moduli is given [6,9]:

$$
{ }^{n+1} \mathbf{c}=\sum_{i=1}^{n_{\text {dimm }}} \sum_{j=1}^{n_{\text {dimm }}}\left[{ }^{n+1} \mathbf{a}\right]_{i j} \mathbf{n}_{\mathrm{tr}}^{i} \otimes \mathbf{n}_{\mathrm{tr}}^{i} \otimes \mathbf{n}_{\mathrm{tr}}^{j} \otimes \mathbf{n}_{\mathrm{tr}}^{i}+2 \sum_{i=1}^{n_{\mathrm{dim}}}\left[{ }^{n+1} \bar{\tau}\right]_{i} \hat{\mathbf{c}}_{\mathrm{tr}}^{i}
$$

where ${ }^{n+1}$ is a matrix of order $n_{\text {dim }}$ defined as

$$
{ }^{n+1} \mathrm{a}=\frac{\mathrm{d}^{n+1} \bar{\tau}}{\mathrm{d} \varepsilon_{\mathrm{ix}}^{e}}
$$

The tensors $\left\{\hat{\mathbf{c}}_{\mathrm{rr}}^{i}\right\}_{i=1, \ldots, n_{\text {dim }}}$ can be expressed on the basis $\left\{\mathbf{n}_{\mathrm{tr}}^{i}\right\}_{i=1, \ldots, n_{\mathrm{dim}}}$ and depend on the values $\varepsilon_{\mathrm{tr}}^{\mathrm{e}}$. Therefore, they are fully determined from the elastic trial state $[6,9]$.

Equation (23) corresponds to the linearization of the Kirchhoff stresses at time ${ }^{n+1} t_{3}{ }^{n+1} \tau$, with respect to the gradient of the incremental displacements. It can be found by applying the chain
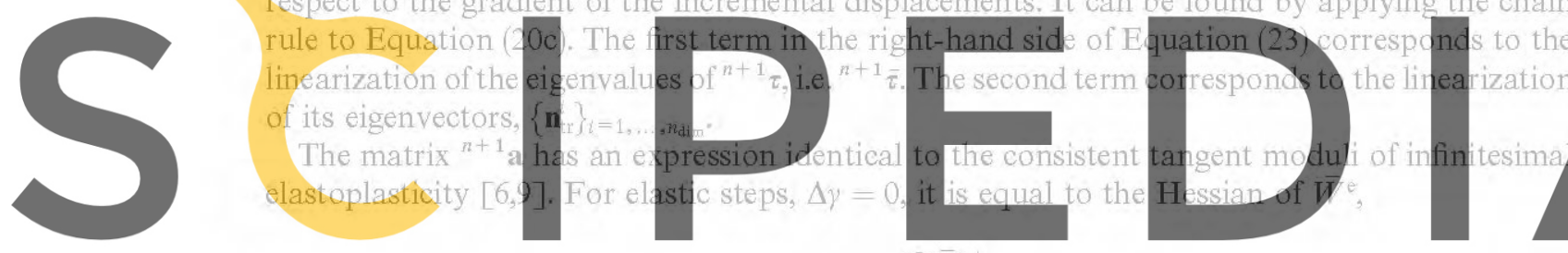

$$
n+1 \mathrm{a}=\frac{\mathrm{d}^{2} \bar{W}^{\mathrm{e}}}{\mathrm{d}^{2}}
$$

Register for free at https//www.scipedia.com to download the version without the watermark

For plastic steps, $\Delta y>0$, it can be expressed as [34]

$$
{ }^{n+1} \mathbf{a}=\mathbf{P}^{\mathrm{T}} \tilde{\mathbf{G}} \mathbf{P}-\frac{\mathbf{P}^{\mathrm{T}} \tilde{\mathbf{G}} \mathbf{m} \nabla f^{\mathrm{T}} \tilde{\mathbf{G}} \mathbf{P}}{\nabla f^{\mathrm{T}} \tilde{\mathbf{G}} \mathbf{m}}
$$

with all quantities evaluated at time ${ }^{n+1} t$ and where the following notation has been introduced:

- $\mathbf{P}$ is a projection matrix of $n_{\mathrm{dim}}+n_{\alpha}$ rows and $n_{\mathrm{dim}}$ columns, $\mathbf{P}^{\mathrm{T}}=\left(\mathbf{I}_{n_{\mathrm{dim}}}, \mathbf{0}_{n_{\mathrm{dim},}, n_{\mathrm{\alpha}}}\right)$, and $\mathbf{0}_{n_{\mathrm{dim},}, n_{\mathrm{\alpha}}}$ is a null matrix of $n_{\text {dim }}$ rows and $n_{\alpha}$ columns (recall that $\alpha \in \mathbb{R}^{n_{\alpha}}$ ).

- $\tilde{\mathbf{G}}$ is a matrix of order $n_{\mathrm{dim}}+n_{\alpha}$ equal to $\left(\mathbf{G}^{-1}+\Delta \gamma \nabla \mathbf{m}\right)^{-1}$, with $\mathbf{G}$ a symmetric matrix defined as

$$
\mathbf{G}=\left(\begin{array}{ll}
\frac{\mathrm{d}^{2} \bar{W}^{\mathrm{e}}}{\mathrm{d} \varepsilon^{\mathrm{e}^{2}}} & 0 \\
0 & \frac{\mathrm{d}^{2} W^{\mathrm{p}}}{\mathrm{d} \boldsymbol{\alpha}^{2}}
\end{array}\right),
$$


- $\mathbf{m}$ is a $n_{\mathrm{dim}}+n_{\alpha}$ vector defined as $\mathbf{m}^{\mathrm{T}}=\left(\mathbf{m}_{\tilde{\tau}}^{\mathrm{T}}, \mathbf{m}_{\mathrm{q}}^{\mathrm{T}}\right)$.

- And $\nabla$ refers to the derivatives with respect to $\bar{\tau}$ and $\mathbf{q}$,

$$
\nabla f=\left(\begin{array}{c}
\frac{\partial f}{\partial \bar{\tau}} \\
\frac{\partial f}{\partial \mathbf{q}}
\end{array}\right) \text { and } \quad \nabla \mathbf{m}=\left(\begin{array}{cc}
\frac{\partial \mathbf{m}_{\bar{\tau}}}{\partial \bar{\tau}} & \frac{\partial \mathbf{m}_{\bar{\tau}}}{\partial \mathbf{q}} \\
\frac{\partial \mathbf{m}_{\mathbf{q}}}{\partial \bar{\tau}} & \frac{\partial \mathbf{m}_{\mathbf{q}}}{\partial \mathbf{q}}
\end{array}\right)
$$

\subsection{Extension to the density-dependent case}

In the following, the consistent linearization of the numerical-time integration scheme applied to density-dependent plastic models is presented. Although the dependence on the density of the plastic equations does not modify the standard integration algorithm, it affects the consistent tangent moduli.

In a plastic step, the relative density, ${ }^{n+1} \eta$, affects the plastic response and thus the value of ${ }^{n+1} \tau$. It depends on the gradient of the incremental displacement through the relation
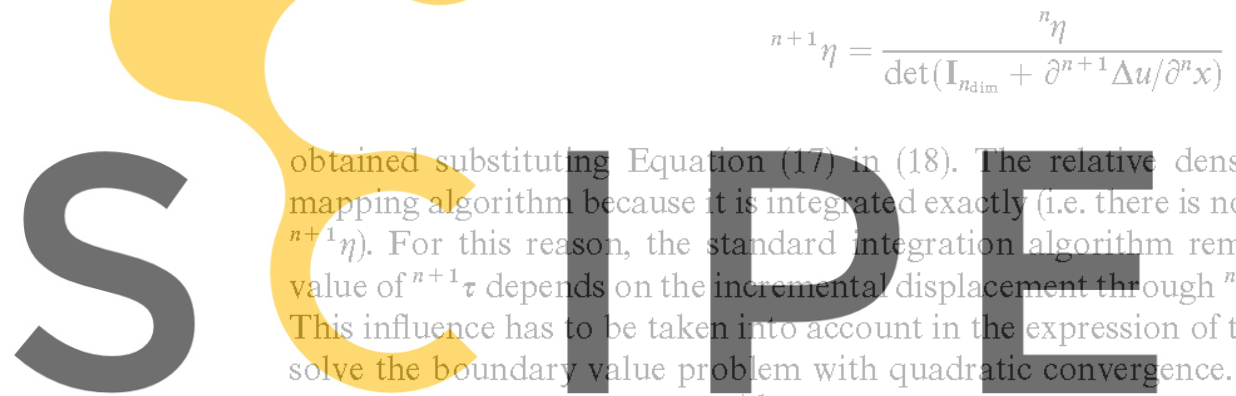

The general expression of

c, Equation (23), is valid for density-dependent plastic models.

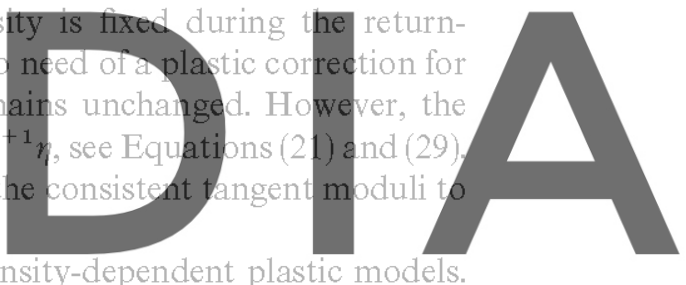

The dependence on the density does not affect the application of the chain rule nor the

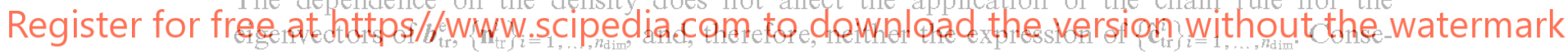
quently, the influence of the density is restricted to the value of ${ }^{n+1} \bar{\tau}$ and the matrix ${ }^{n+1}$ a. The value of ${ }^{n+1} \bar{\tau}$ is obtained directly from the numerical time-integration scheme, Equation (21). On the other hand, ${ }^{n+1} \mathbf{a}$ only depends on the trial state for elastic steps. In summary, only the new expression of ${ }^{n+1}$ a for plastic steps must be obtained to extend Equation (23) to densitydependent plastic models.

In the following, the consistent linearization of the plastic corrector step is presented. It is shown that the new, more general, expression of the moduli ${ }^{n+1} \mathbf{a}$ is composed of two terms, one equal to the expression for density-independent models, Equation (26), and another one which adds the influence of the density. In order to do that it is useful to rephrase the dependence of ${ }^{n+1} \eta$ on ${ }^{n+1} f$, Equation (18), as

$$
{ }^{n+1} \eta={ }^{n} \hat{\eta} \exp \left(-\operatorname{tr}\left(\varepsilon_{\mathrm{tr}}^{\mathrm{e}}\right)\right)
$$

with ${ }^{n} \hat{\eta}={ }^{n} \eta \sqrt{\operatorname{det}\left({ }^{n} b^{\mathrm{e}}\right)}$ a known value from the previous time step and where $\operatorname{tr}(*)$ means trace of * (that is, $\sum_{i=1}^{n_{\text {dim }}}[*]_{i}$ ). Equation (30) is found by applying the determinant function to both sides of Equation (19) and substituting Equation (20a) into it. 
From the definition of ${ }^{n+1}$ a, Equation (24), and the application of the chain rule a new more comvenient expression of ${ }^{n+1} \mathbf{a}$ is found:

$$
{ }^{n+1} \mathbf{a}=\frac{\mathrm{d}^{n+1} \bar{\tau}}{\mathrm{d}^{n+1} \varepsilon^{\mathrm{e}}} \frac{\mathrm{d}^{n+1} \varepsilon^{\mathrm{e}}}{\mathrm{d} \varepsilon_{\mathrm{tr}}^{\mathrm{e}}}=\left.\frac{\mathrm{d}^{2} \bar{W}^{\mathrm{e}}}{\mathrm{d} \varepsilon^{\mathrm{e}^{2}}}\right|_{t={ }^{n+1} t} \frac{\mathrm{d}^{n+1} \varepsilon^{\mathrm{e}}}{\mathrm{d} \varepsilon_{\mathrm{tr}}^{\mathrm{e}}}
$$

Equation (31) shows that ${ }^{n+1} \mathbf{a}$ is determined once the total influence of $\varepsilon_{\mathrm{tr}}^{\mathrm{e}}$ on ${ }^{n+1} \varepsilon^{\mathrm{e}}$ is found. This influence is given by the the non-linear system of Equations (21) and the relationship between ${ }^{n+1} \eta$ and $\varepsilon_{\mathrm{tr}}^{\mathrm{e}}$, Equation (30). Thus, it can be computed by linearizing the non-linear system of equations

$$
\begin{aligned}
{ }^{n+1} \varepsilon^{\mathrm{e}}+\Delta \gamma \mathbf{m}_{\bar{\tau}}\left({ }^{n+1} \bar{\tau},{ }^{n+1} \mathbf{q}^{n+1} \eta\right) & =\varepsilon_{\mathrm{tr}}^{\mathrm{e}} \\
-{ }^{n+1} \boldsymbol{\alpha}+\Delta \gamma \mathbf{m}_{\mathrm{q}}\left({ }^{n+1} \bar{\tau}^{n+1} \mathbf{q}^{n+1} \eta\right) & =-\boldsymbol{\alpha}_{\mathrm{tr}} \\
f\left({ }^{n+1} \bar{\tau},{ }^{n+1} \mathrm{q}_{2}{ }^{n+1} \eta\right) & =0 \\
{ }^{n+1} \eta & ={ }^{n} \eta \exp \left(-\operatorname{tr}\left(\varepsilon_{\mathrm{tr}}^{\mathrm{e}}\right)\right)
\end{aligned}
$$

\section{and relationships (22) and (4b), this links ${ }^{n+1} \bar{\tau}$ and ${ }^{n+1} q$ with ${ }^{n+1} \xi^{e}$ and ${ }^{n+1} \alpha$.}

The linearization of Equations (32) reads, in compact matrix form
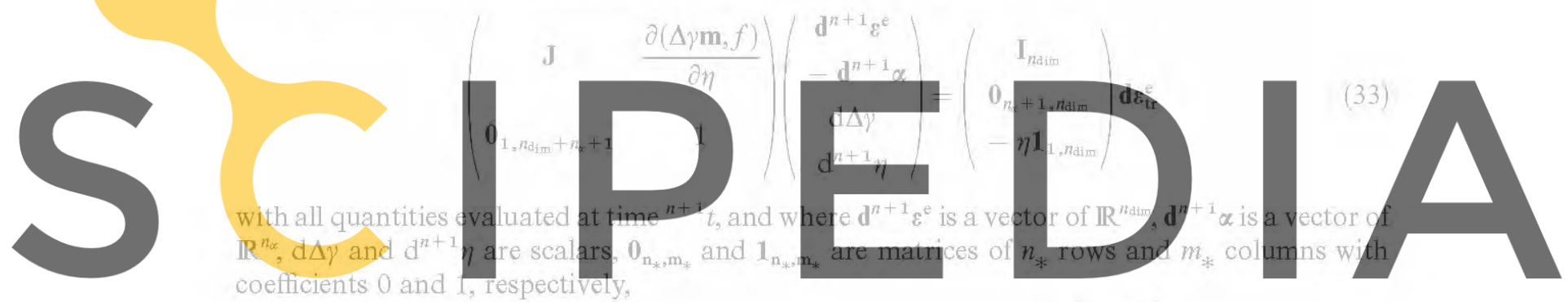

Register for free at https//wwwiscipedia.G@m to download the version without the watermark $J=\left(\begin{array}{ll}\mathrm{J} \\ \nabla f^{\mathrm{T}} \mathrm{G}\end{array}\right.$

From Equation (33) and the relationship

$$
\mathbf{d}^{n+1} \varepsilon^{e}=\frac{\mathrm{d}^{n+1} \varepsilon^{e}}{\mathrm{~d} \varepsilon_{\mathrm{tr}}^{e}} \mathbf{d} \varepsilon_{t r}^{e}
$$

it results that

$$
\frac{\mathrm{d}^{n+1} \boldsymbol{\varepsilon}^{\mathrm{e}}}{\mathrm{d} \boldsymbol{\varepsilon}_{\mathrm{tr}}^{\mathrm{e}}}=\left(\begin{array}{lll}
\mathbf{P}^{\mathrm{T}} & \mathbf{0}_{n_{\mathrm{dim}, \mathrm{I}} \mathrm{I}} & \mathbf{0}_{n_{\mathrm{dim}, \mathrm{I}}, \mathrm{I}}
\end{array}\right)\left(\begin{array}{cc}
\mathbf{J} & \frac{\partial(\Delta \gamma \mathbf{m}, f)}{\partial \eta} \\
\mathbf{0}_{1, n_{\mathrm{dim}}+n_{\mathrm{\alpha}}+1} & 1
\end{array}\right)\left(\begin{array}{c}
\mathbf{P} \\
\mathbf{0}_{1, n_{\mathrm{dim}}} \\
-\eta \mathbf{1}_{1, n_{\mathrm{ditm}}}
\end{array}\right)
$$

which is equivalent to

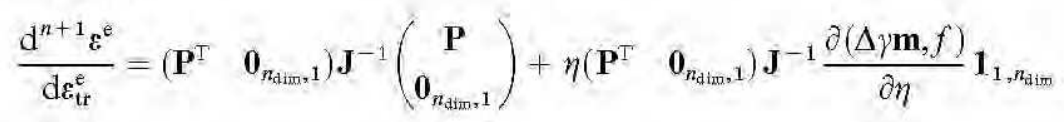


The expression of ${ }^{n+1} \mathbf{a}$ is found substituting Equation (37) into Equation (31). It has two parts, one for each term on the right-hand side of Equation (37),

$$
{ }^{n+1} \mathbf{a}={ }^{n+1} \mathbf{a}_{\eta-\text { indep }}^{\text {ep }}+{ }^{n+1} \mathbf{a}_{\eta}^{\text {ep }}
$$

The first part, ${ }^{n+1} \mathbf{a}_{\eta \text {-indep }}^{\text {ep }}$, is equal to the standard one for density-independent plastic models, Equation (26). The second part, ${ }^{n+1} \mathbf{a}_{\eta}^{\text {ep }}$, takes into account the influence of the density. After some manipulations it can be expressed as

$$
{ }^{n+1} \mathbf{a}_{\eta}^{\mathrm{ep}}=\eta \Delta \gamma \mathbf{P}^{\mathrm{T}}\left(\tilde{\mathbf{G}}-\frac{\tilde{\mathbf{G}} \mathbf{m} \nabla f \tilde{\mathbf{G}}}{\nabla f^{\mathrm{T}} \tilde{\mathbf{G}} \mathbf{m}}\right) \frac{\partial \mathbf{m}}{\partial \eta} \mathbf{1}_{1, n_{\mathrm{dim}}}+\eta \frac{\partial f}{\partial \eta} \mathbf{P}^{\mathrm{T}} \frac{\tilde{\mathbf{G}} \mathbf{m}}{\nabla f^{\mathrm{T}} \tilde{\mathbf{G}} \mathbf{m}} \mathbf{1}_{1, n_{\mathrm{dim}}}
$$

In the density-independent case, symmetric tangent moduli are obtained for associative material models, $m=\nabla f$, see Equation (15). On the contrary, unsymmetric moduli are found with all density-dependent material models because ${ }^{n+1} \mathrm{a}_{\eta}^{\mathrm{ep}}$ is unsymmetric except for very particular problems (see Section 4). For this reason, in density-dependent plasticity, unsymmetric linear solvers have to be used in order to keep the characteristic quadratic convergence of the Newton-Raphson method.

On the other hand, it is important to remark that the expression of ${ }^{n+1}$ a for density-dependent plastic models can be computed with just a few more matrix-vector products than the standard one for density-independent plastic models (cf. Equations (39) and (26)), and, as expected, the
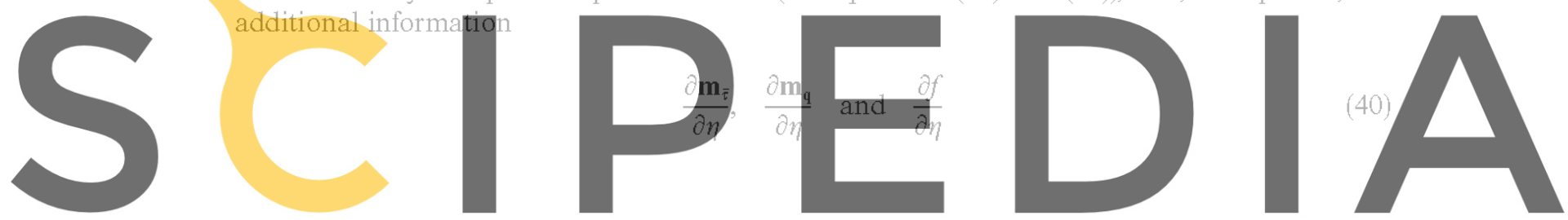

Register for free at https//www.scipedia.com to download the version without the watermark with density-dependent plastic models. The consistent tangent moduli presented in the previous section are used.

Examples with three elastoplastic models are presented: a Drucker-Prager model, an elliptic model and a cone-cap model. The three models have no internal variables, $n_{\alpha}=0$, a usual feature in the density-dependent models found in the literature. This fact simplifies some equations presented in the previous section. However, the structure and the dependence on the density of the consistent tangent moduli remain unchanged, see Equations (26) and (39), and, therefore, the examples are fully representative.

The three plastic models are complemented with Hencky's hyperelastic law, which leads to a linear relationship between $\bar{\tau}$ and $\varepsilon^{e}$ equal to that of linear elasticity between stresses and small strains. The use of other hyperelastic laws is straightforward. The main features of the three models are described in this section. A more detailed presentation is included in the appendix. Two invariants of the stresses are used to show some results. They are also defined in the appendix.

The examples with the Drucker-Prager model show that the proposed approach (numerical time-integration and consistent tangent moduli) is valid for Cauchy-based elastoplastic models. 
The examples with the elliptic and the cone-cap models show that the consistent tangent moduli also give quadratic convergence for general density-dependent constitutive laws. The yield function and the flow vector of the three models are expressed as functions of the Kirchhoff stresses and the relative density, as presented in Section 2.

Elliptic models are widely used in porous material modelling, see Reference [27] and references therein, and specifically in powder compaction simulations, see References $[21,22,26]$. The formulation used in this work is based on that presented by Oliver et al. [21,22]. In these references, the elastic strains are assumed small and the kinematic description of the model is simplified. A specific return mapping algorithm and the corresponding consistent tangent moduli are presented. In this work, the general finite hyperelastoplasticity framework is used. The results of both approaches are compared in some introductory examples. After that, a more demanding example is solved using the arbitrary Lagrangian-Eulerian formulation for multiplicative elastoplasticity [32]. Quadratic converge is obtained in all cases.

The cone-cap model is an extension of the elliptic model. It is defined by a density-dependent Drucker-Prager yield surface and a non-centred ellipse. The yield function and the flow rule are similar to other cone-cap models used recently in powder compaction simulations, see for instance References [23,24,28-31]. However, the cone-cap model used here is defined, like the elliptic model, in the general finite hyperelastoplasticity framework presented in Section 2, without kinematic simplifying assumptions. The results obtained in a particular example are compared with those of the elliptic model. Quadratic convergence results are also shown.

Remark 3. In the examples involving the elliptic and the cone-cap models, the derivatives of the yield function and the flow vector, Equations (40), are computed with a first-order numerical approximation, as proposed in Reference [35] for flow vector derivatives in a small strain context. Also in the present application, numerical differentiation does not disturb the quadratic convergence of the Newton-Raphson method. Convergence is checked with the relative error in energy norm. A strict tolerance of $10^{-10}$ has been used in all the examples in order to show clearly the quadratic convergence. Quite larger values may be chosen in practice.

\subsection{Drucker-Prager model simulations}

The following examples involve a Cauchy-based Drucker-Prager model (CDP). They illustrate that the proposed approach for the numerical time-integration, standard return-mapping algorithm, and the new consistent tangent moduli, equation (38), are valid for Cauchy-based elastoplastic models. The model is presented in the appendix, Equation (A6).

Simulations with the standard density-independent Kirchhoff-based Drucker-Prager model (KDP), Equation (A4), are also included for comparative purposes. The flow vectors of both models, CDP and KDP, are, respectively, equal to the partial derivative of the corresponding yield function with respect to the Kirchhoff stresses. The two models are completely different from a modelling point of view, because different stress measures are used, so qualitatively different results are expected. With the KDP model, the plastic material behaviour is assumed to be density-independent. With the CDP model, on the other hand, density dependence is accounted for in an indirect fashion, because $\sigma$ depends both on $\eta$ and $\tau$, see Equation 12. See Reference [20] for a discussion on the role of the stress measure in the plastic equations.

A square of $1 \mathrm{~cm}$ of length is subjected to a plane strain uniaxial compression test [20]. The domain is modelled by a single bilinear element. A height reduction of 75 per cent is imposed with five increments of 1 per cet and 14 increments of 5 per cent. The material parameters are 


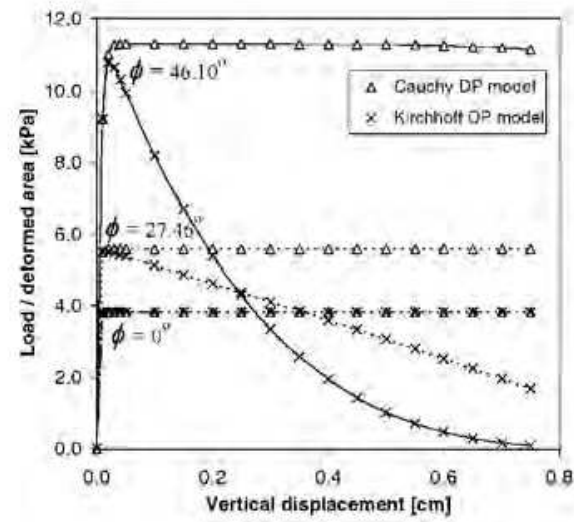

(a)

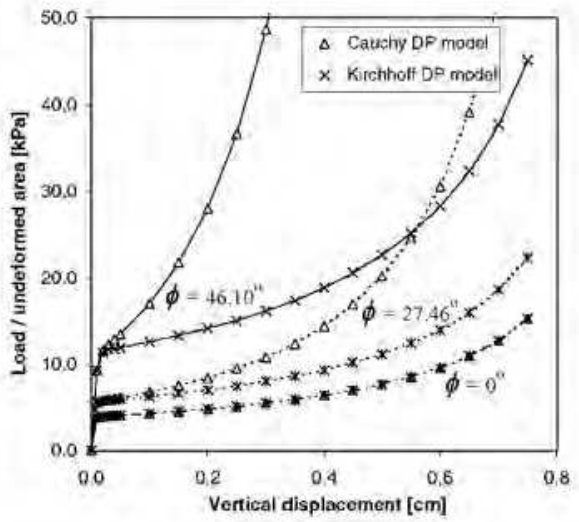

(b)

Figure 1. Load-displacement results of the uniaxial compression test. Cauchy-and Kirchhoffbased Drucker-Prager models with different friction angles, $\phi$. (a) Load divided by deformed area and (b) load divided by undeformed area.

a Young's modulus, $E$, equal to $10 \mathrm{GPa}$, a Poisson's ratio, $v$, equal to 0.3 , and a cohesion of $2.7 \sqrt{3 / 2} \mathrm{GPa}$. The results for three values of the friction angle, $\phi=0,27.46$ and 46.1 are computed [20].

The curves of load divided by deformed and undeformed area versus vertical displacement are depicted in Figure 1. The KDP model with friction angles greater than $0^{\circ}$ exhibits a post-peak softening in the load divided by deformed area-vertical displacement relationship. The softening increases with the friction angle. As Meschke and Liu [20] indicate, this behaviour is related with the inelastic volume change produced by the flow rule. On the other hand, the results of both models, KDP and CDP, for $\phi=0^{\circ}$ differ only on the elastic volume change, which is not significant for the chosen material parameters.

The convergence results of both models for different vertical displacements and the three friction angles are depicted in Figure 2. All results are quadratic. The influence of neglecting the density contribution on the consistent tangent moduli is shown in Figure 3. The convergence results for several load increments and the CDP model with a friction angle of $46.1^{\circ}$ are depicted. In this case, non-convergence is detected after a vertical displacement of $0.65 \mathrm{~cm}$. In all convergent load increments. after the initial two or three iterations, the convergence is clearly linear. The influence of the density on the consistent tangent moduli is evident comparing Figures 3 and $2(\mathrm{f})$.

\subsection{Elliptic model simulations}

In the following, some examples of density-dependent multiplicative elastoplasticity with an elliptic yield function are analysed. The yield function expression is presented in the appendix. Its traces on the meridian plane $q_{\bar{z}}-p_{\bar{z}}$ for different relative densities, $\eta$ and a particular set of material parameters are depicted in Figure 4 . The flow vector is equal to the partial derivatives of the yield function with respect to the Kirchhoff stresses. Two sets of material parameters are used. Their values are summarized in Table I. Both are calibrated in Reference [22] by comparing the results of numerical simulations with experiments. 

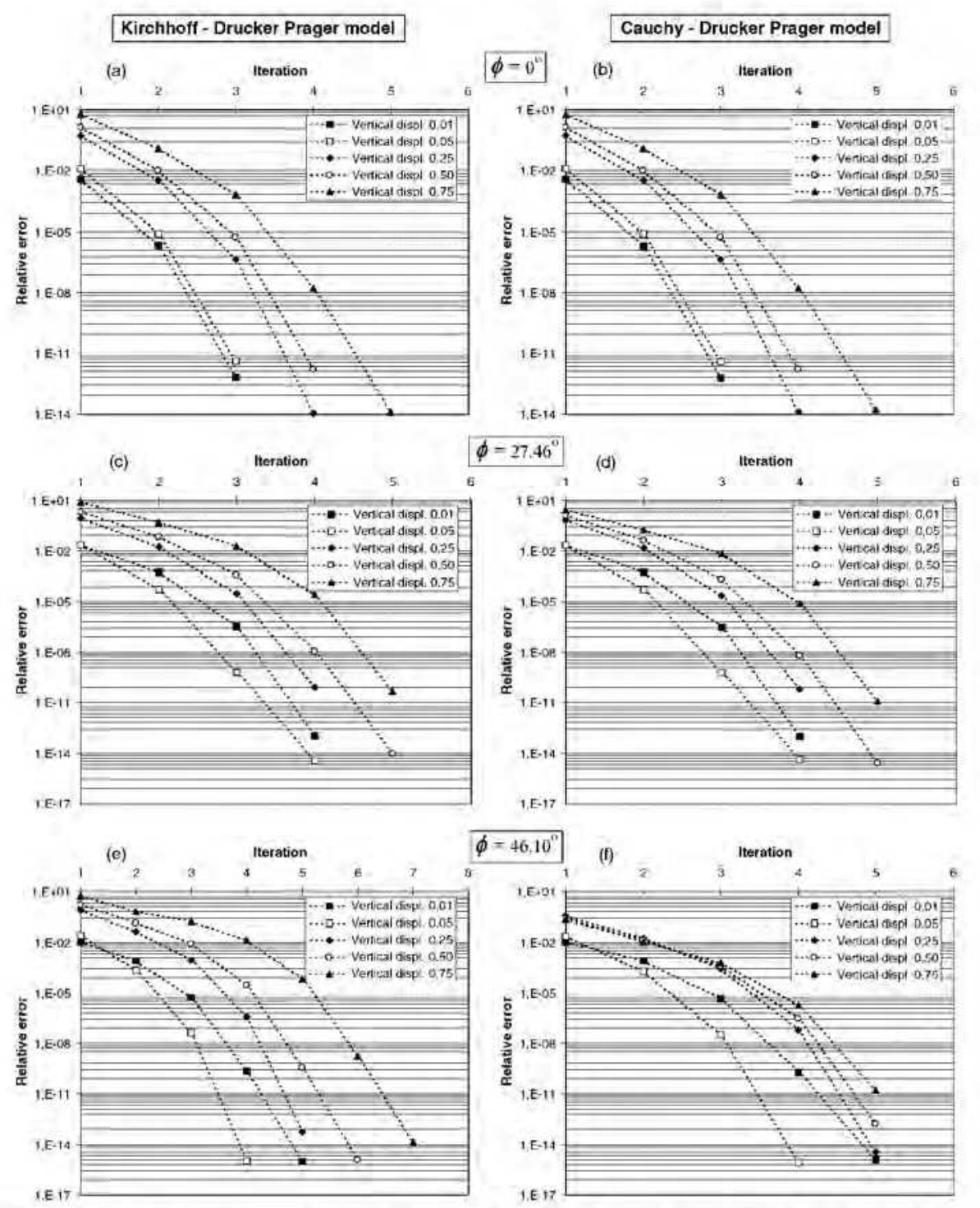

Figure 2. Uniaxial compression test. Left (a,c,e) Kirchhoff-based and right (b,d,f) Cauchy-based Drucker-Prager models with different friction angles. $\phi$. Convergence results for different vertical displacements.

The first set of compaction experiments [36] is modelled in Reference [22] with powder-A parameters. A sample with an initial height of $24 \mathrm{~mm}$ and a diameter of $20 \mathrm{~mm}$ was used. Both isostatic compaction and triaxial tests were performed. The triaxial tests consisted of an initial isostatic compaction step up to a pressure of 50,150 or $400 \mathrm{MPa}$, followed by an uniaxial compaction up to a vertical pressure of $1250 \mathrm{MPa}$ (keeping the external radial pressure constant). The problem is axisymmetric. The sample is modelled by a $2 \mathrm{D}$ structured mesh of 100 bilinear 


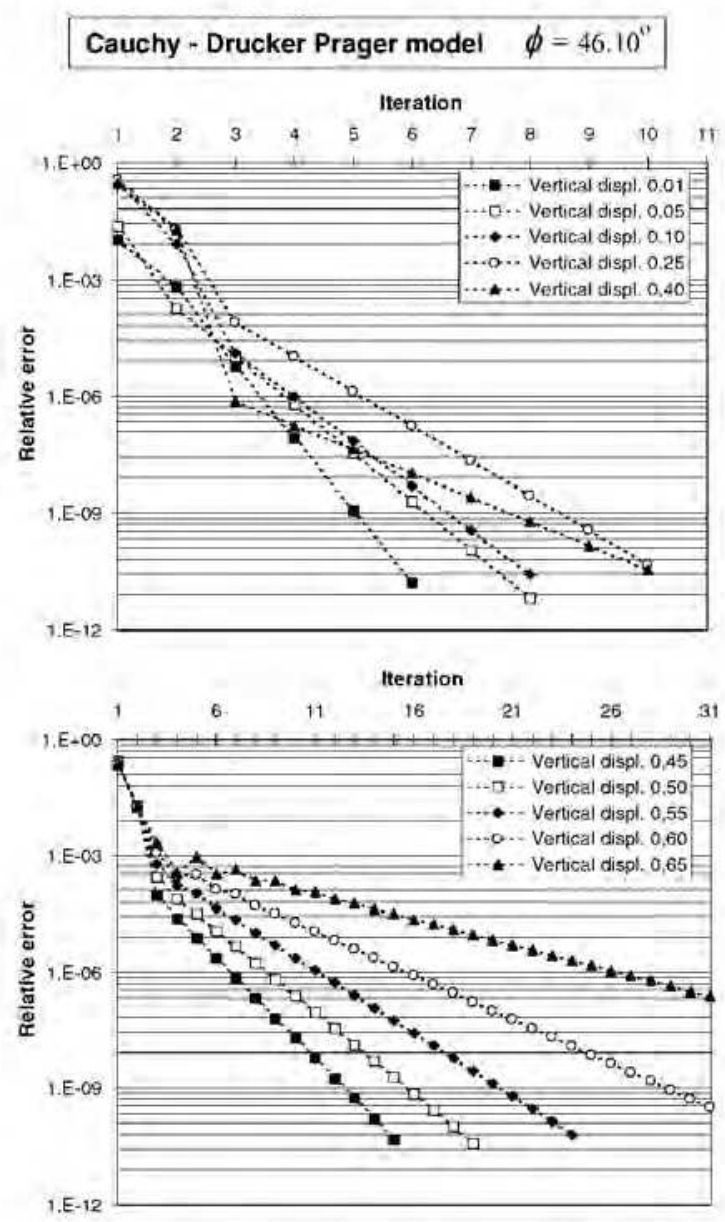

Figure 3. Convergence results for different vertical displacements obtained with the standard (densityindependent) consistent tangent moduli, ${ }^{n+1} \mathbf{a}={ }^{n+1} \mathbf{a}_{n}^{\mathrm{ep}}$-indep.

elements. The friction effects are not considered [22]. The results of the numerical simulations and the experiments are compared in Figure 5. The results of the simulations agree with the experimental ones and the results presented in Reference [22], not shown in Figure 5.

The convergence results for different load levels of the isostatic test are depicted in Figure 6. Quadratic convergence is found for all load increments. However, if a consistent tangent matrix for density-independent problems is used divergence is found at the first load increment. This example is more demanding in the initial load levels than in the last ones. This fact is in agreement with the dependence of the density on the applied pressure, see Figure 5(a). At the beginning of the test, a given increment of the pressure causes a larger variation of the density than at the end of the test.

The convergence results for the triaxial test with an initial isostatic pressure of $150 \mathrm{MPa}$ are summarized in Figure 7. Results for different load levels of the isostatic and the uniaxial 


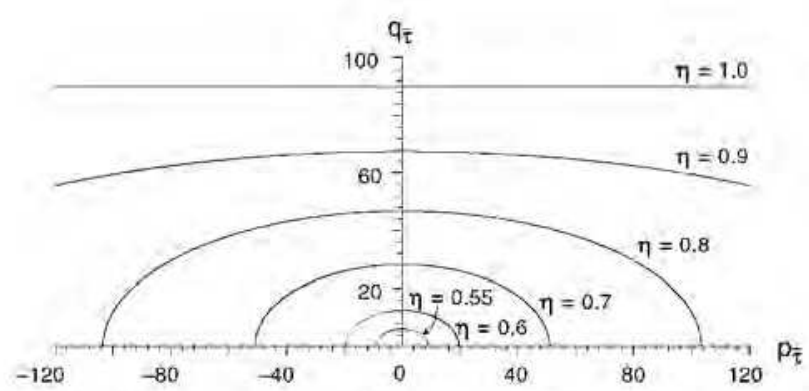

Figure 4. Traces of the elliptic yield function on the meridian plane $q_{\tau}-p_{t}$ for different relative densities, $\eta$. Powder-A material parameters, see Table I.

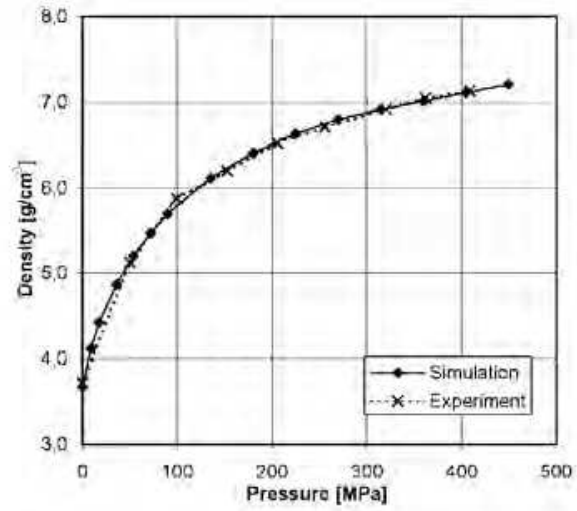

(a)

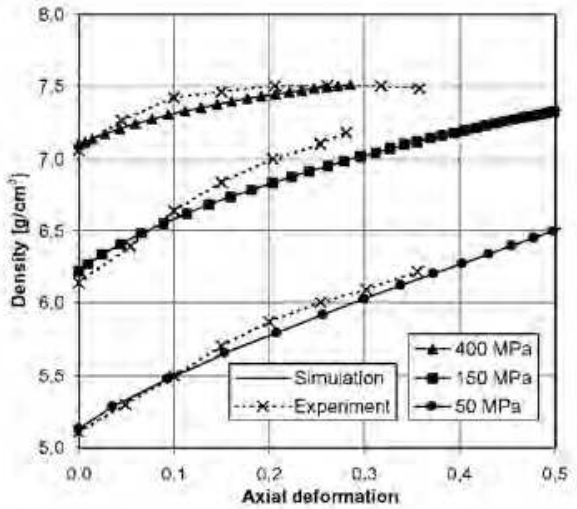

(b)

Figure 5. Results of (a) isostatic and (b) triaxial tests with powder-A material, see Table L. Experimental data from Doremus et al. [36].

compression parts of the test are depicted. The isostatic part is solved with 52 non-uniform increments ( 4 of 1 per cent, 48 of 2 per cent) and the uniaxial part with 20 equal increments. The results obtained using an unsymmetric solver for the linear systems of equations (recall that the full consistent tangent moduli ${ }^{n+1} \mathbf{a}$ is not symmetric) and a symmetric solver (with the symmetrized part of ${ }^{n+1}$ a) are shown. As expected, the results obtained with the unsymmetric consistent tangent moduli are quadratic, see Figures 7(a) and 7(c). Remarkably, the convergence results obtained with the symmetric solver during the isostatic part of the test are also quadratic, see Figure 7(b). This is due to the pure hydrostatic character of the test, which leads to a symmetric consistent tangent matrix, see Equation (39). In the uniaxial compression part of the test the symmetric solver gives linear convergence, Figure 7(d). The same influence on the convergence has been reported for other problems with unsymmetric consistent tangent matrices solved with a linear solver for symmetric matrices [20].

The second set of compaction experiments [37] is modelled in Reference [22] with powder-B parameters of Table I. A sample with an initial height of $80 \mathrm{~mm}$ and a diameter of $14.3 \mathrm{~mm}$ was used. Both isostatic and uniaxial compaction tests were performed. The sample is modelled by 


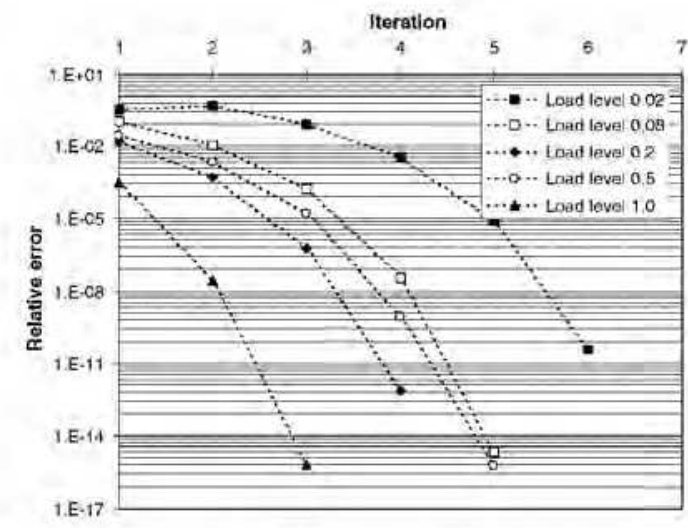

Figure 6. Powder-A isostatic test. Convergence results for different load levels.

Unsymmetric linear solver

(a) Isastatic compression

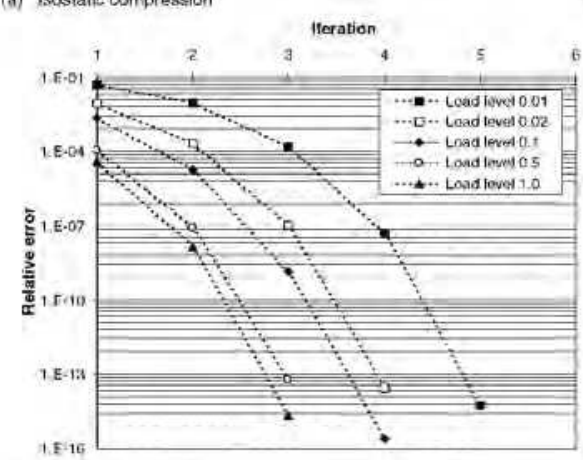

(c) Uniaxial compression

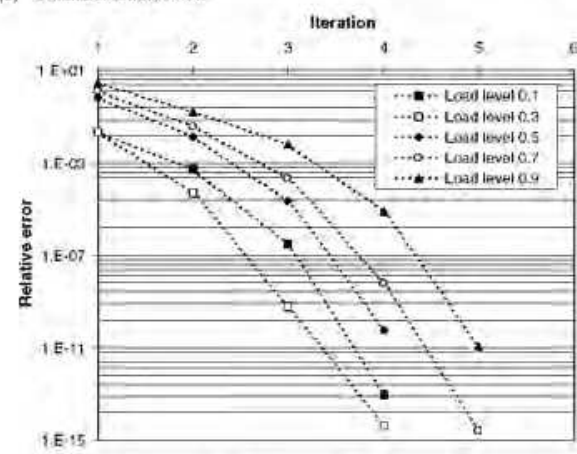

Symmetric linear solver

(b) Isostallic compression

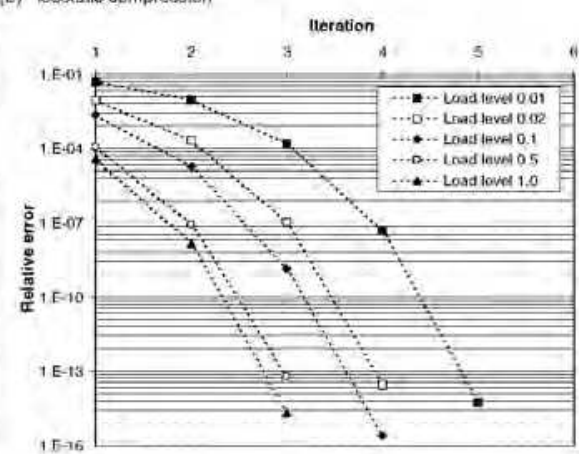

(d) Uniaxial compression

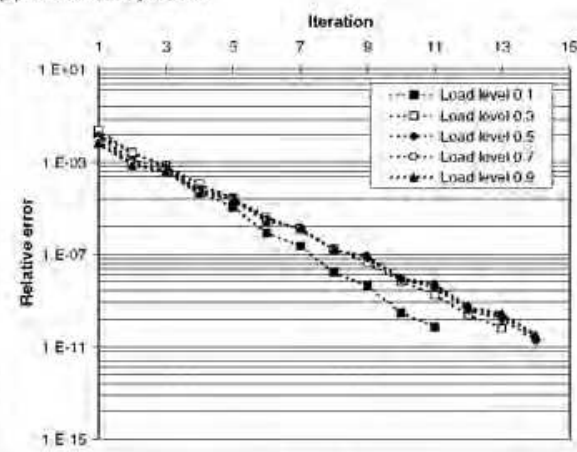

Figure 7. Powder- $\Lambda$ triaxial test (150 MPa initial isostatic compaction). Convergence results for different load levels and with $(a, c)$ unsymmetric and $(b, d)$ symmetric linear solvers, left and right, respectively. 


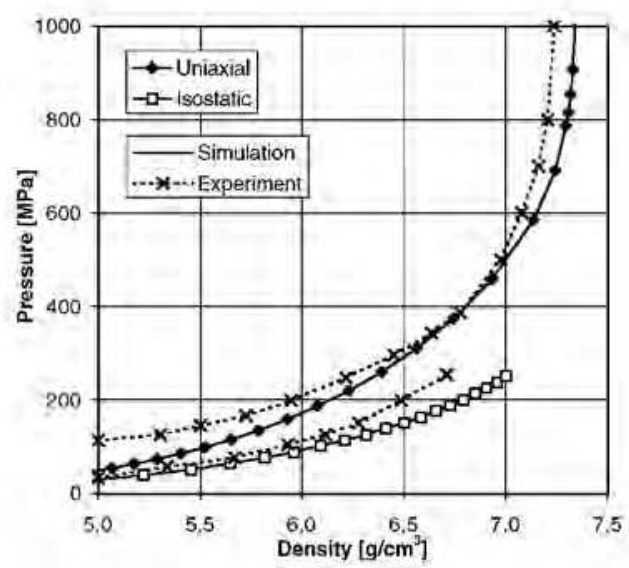

Figure 8. Results of isostatic and uniaxial tests with powder-B material, see Table I. Experimental data from Ernst and Barnekov [37].

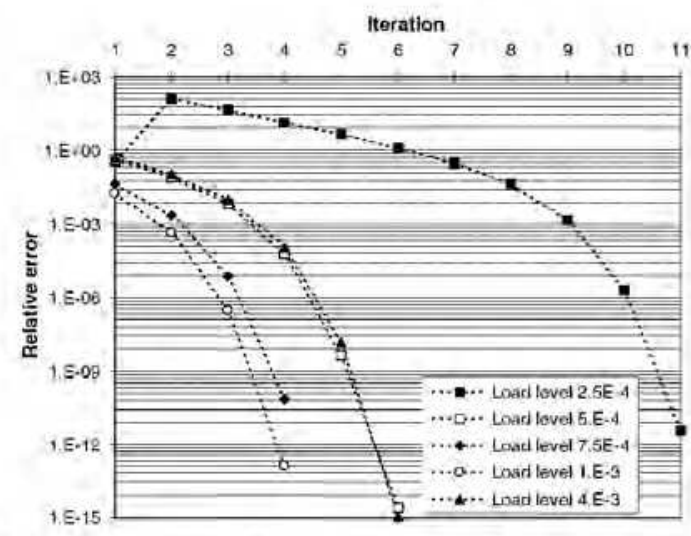

Figure 9. Powder-B isostatic test. Convergence results for different load levels.

a structured mesh of 100 bilinear elements, and no friction effects are considered. The results of the numerical simulations and the experiments are compared in Figure 8. The numerical results are in general agreement with the experimental results (recall that the material parameters have not been re-calibrated, they are the same used in Reference [22] for this example). They are also in agreement with the numerical results presented in Reference [22], not shown in Figure 8. The main difference is that the density predicted in this work is a little bit higher than the one shown in Reference [22]. This difference may be related with the large elastic strains, which in the present formulation are taken into account.

The convergence results for different load levels of the initial part of the isostatic test are depicted in Figure 9. Small load increments are required at the beginning of the test to ensure convergence of the boundary value problem ( 4 increments of 0.025 per cent and 3 increments of 
0.3 per cent of a total load of $250 \mathrm{MPa}$ ). After these initial steps, the load increments are quite larger ( 2 increments of 2 per cent and 19 of 5 per cent). Quadratic convergence is found for all steps.

The last example with the elliptic model is the frictionless compaction of a rotational flanged component. The component is modelled by an axisymmetric representation as illustrated in Figure 10 [29,31]. Powder-A material parameters of Table I are used. This example shows that quadratic convergence is also found in highly demanding boundary value problems.

In the simulation presented in this section the friction effects are neglected. Therefore, the results are a qualitative approximation to the real compaction process, where the friction effects have to be taken into account $[29,31]$. Complete compaction simulations with this material model and friction can be found in Reference [25].

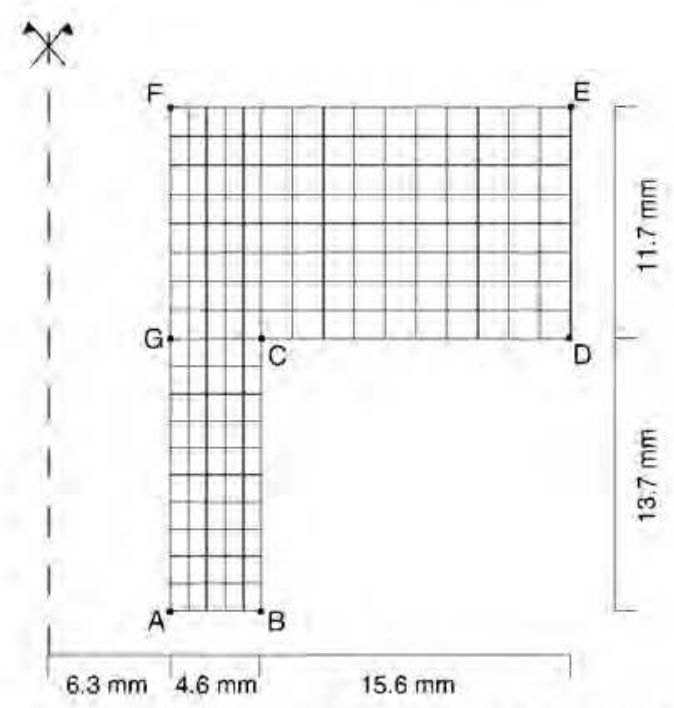

Figure 10. Flanged component. Problem definition (after Lewis and Khoei [29]) and computational mesh.

Table I. Sets of material parameters: $\left(E, v, \sigma_{y}, \eta_{0}, n_{1}, n_{2}\right)$ for the elliptic model and $\left(E, v, \sigma_{y}, \eta_{0}, n_{1}, n_{2}, \phi_{\operatorname{mim}}, \phi_{\max }, C_{\text {ref }}, n_{\mathrm{e}}\right)$ for the cone-cap model.

\begin{tabular}{lll}
\hline & Powder-A & Powder-B \\
\hline$E$ & $2000 \mathrm{MPa}$ & $50000 \mathrm{MPa}$ \\
$v$ & 0.37 & 0.37 \\
$\sigma_{r}$ & $90 \mathrm{MPa}$ & $170 \mathrm{MPa}$ \\
$\eta_{0}$ & 0.489 & 0.4 \\
$n_{1}$ & 1 & 0.3 \\
$n_{2}$ & 2.7 & 5.4 \\
$\phi_{\min }$ & $45^{\circ}$ & - \\
$\phi_{\max }$ & $60^{\circ}$ & - \\
$C_{\text {ref }}$ & $15 \mathrm{MPa}$ & - \\
$n_{\mathrm{c}}$ & 2 & - \\
\hline
\end{tabular}


A structured mesh of 170 eight-noded elements with reduced integration (four Gauss-points per element) is used. The radial displacement of segments $\overline{\mathrm{BC}}, \overline{\mathrm{DE}}$ and $\overline{\mathrm{FA}}$ and the vertical movement of segments $\overline{\mathrm{AB}}$ and $\overline{\mathrm{CD}}$ are set equal to zero (see Figure 10). A vertical movement of $-6.06 \mathrm{~mm}$ is imposed to the segment $\overline{\mathrm{EF}}$ in 80 non-uniform increments. Small increments are needed at the beginning of the test ( 10 of 0.01 per cent, 19 of 0.1 per cent, 2 of 1 per cent) in order to ensure convergence of the boundary value problem. Larger increments are used for the rest of the test (48 of 2 per cent). This is directly related with the high curvature of the yield surface for $\eta$ close to $\eta_{0}$, see Figure 4.

The arbitrary Lagrangian-Eulerian formulation presented in Reference [32] for multiplicative elastoplasticity is used to reduce the mesh distortion. The mesh region ABCG is Eulerian, and equal height elements are prescribed in the mesh region CDEFG. Material parameters are those of powder-A in Table I.

The final distribution of the relative density is shown in Figure 11. The results are qualitatively similar to those presented by Lewis and Khoei [29], although the lack of friction produces a relatively high compacted region near point $\mathrm{F}$ which is not reported in similar test simulations. The convergence results for different load levels are depicted in Figure 12. Quadratic convergence is found in all cases.

The final distribution of Cauchy stresses at Gauss-point level on the meridian plane $q_{\bar{\sigma}}-p_{\bar{\sigma}}$ is depicted in Figure 13. This figure will be useful for comparing the results obtained with this model with those of the cone-cap simulation. During the major part of the load process all Gauss points are in plastic load, dark marks; however at the end of the test a small number of Gauss points, 6 , are under elastic load, light marks. On the other hand, the Gauss points subjected to higher and lower Cauchy mean pressure are located respectively above and below point $\mathrm{C}$.

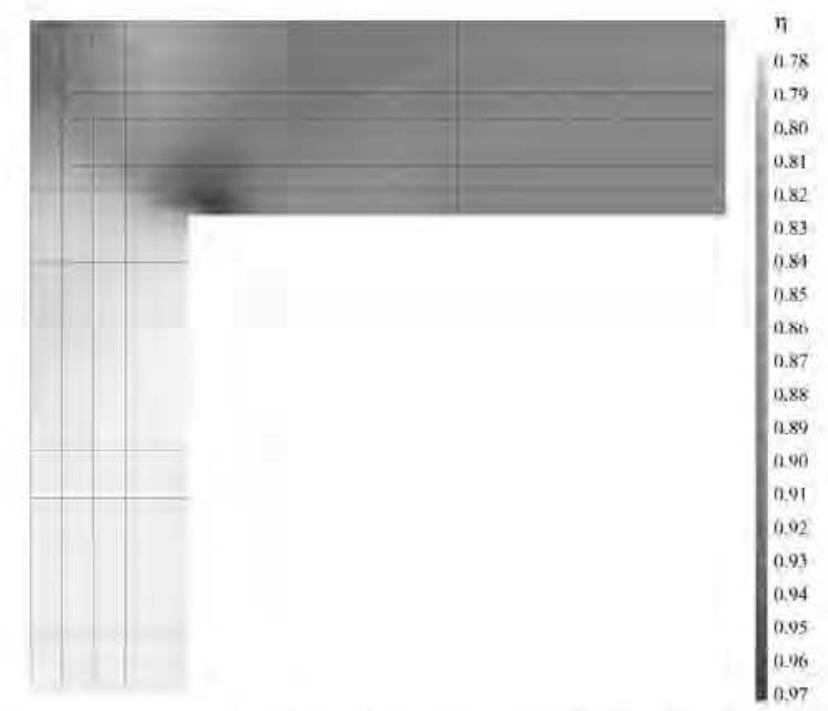

Figure 11. Frictionless compaction of the flanged component with the elliptic model. Final relative density, $\eta$, distribution after a top displacement of $6.06 \mathrm{~mm}$. 


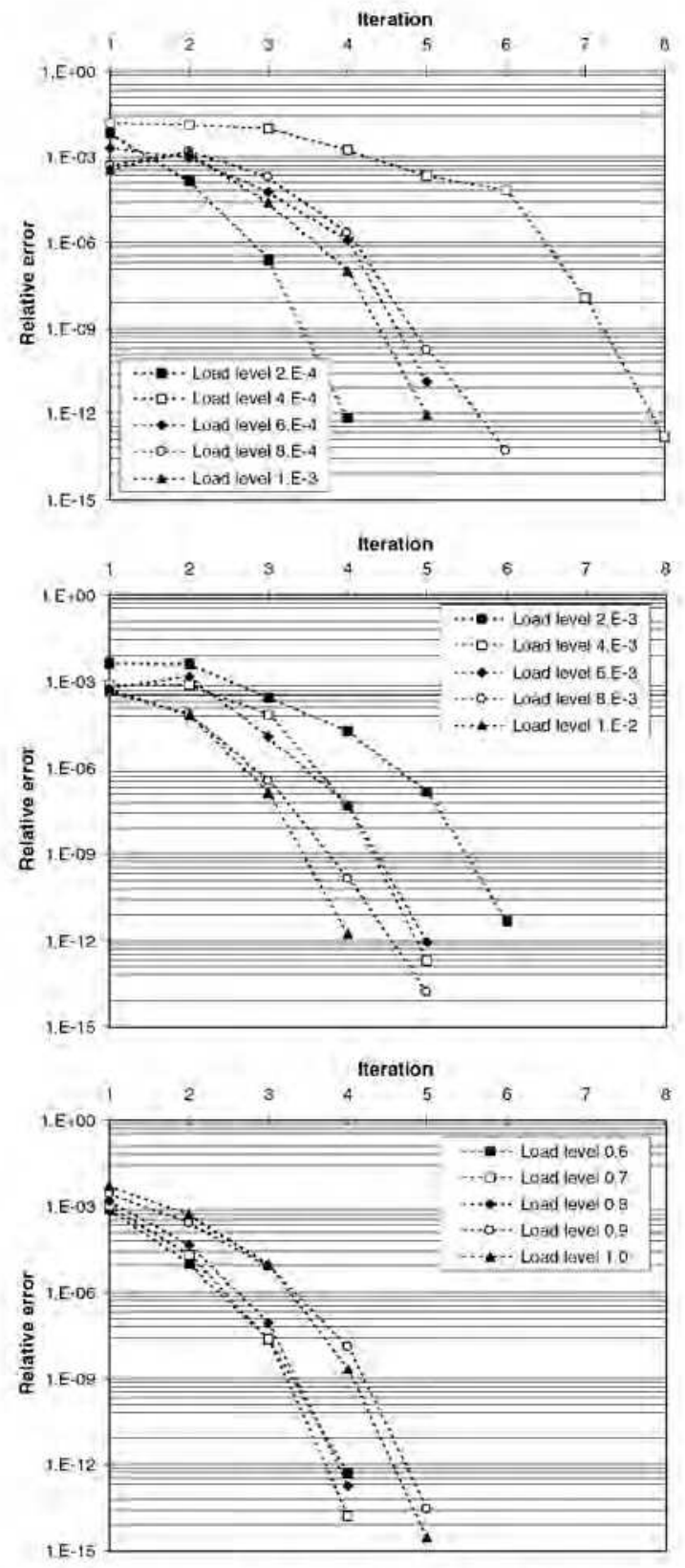

Figure 12. Frictionless compaction of the flanged component with the elliptic model. Convergence results for different load levels. 


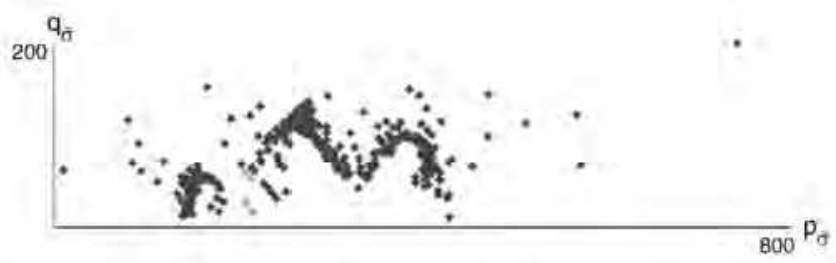

Figure 13. Frictionless compaction of the flanged component with the elliptic model. Final distribution of Cauchy stresses at Gauss-point level on the meridian plane $q_{\sigma}-p_{\bar{\sigma}}$. Dark diamonds indicate plastic steps and light diamonds indicate elastic steps.

\subsection{Cone-cap model simulations}

In the following, the frictionless compaction of a flanged component (Figure 10) is modelled with a cone-cap model. The traces of the yield function on the meridian plane $q_{\mathrm{z}}-p_{\mathrm{t}}$ for different relative densities are depicted in Figure 14. Powder-A material parameters for this model are summarized in Table I. The dependence on density of the cap yield function is similar to that of the elliptic model, cl. Figures 14 and 4 . The cone is defined by a cohesion which increases up to $C_{\text {ref }}$ for $\eta=1$, and a friction angle which varies parabolically from $\phi_{\min }$ at $\eta=\eta_{0}$ to $\phi_{\max }$ at $\eta=1$. The friction angle range and the dependence on the density has been set following [38], with the aim of illustrating the convergence properties of the presented consistent tangent moduli. Lower constant (i.e. density-independent) values are used in Reference [29], and higher constant values are reported in Reference [39].

The flow vector is defined so there are no grey regions on the stress space [40] and therefore no corner return-mapping algorithms $[3,24,41,42]$ are needed. Complete expressions of plastic equations are presented in the appendix. Note that this model represents a more demanding test for the consistent tangent moduli than the elliptic model because the dependence on the density of the yield function and the flow rule is more complicate.

The numerical parameters of the simulation are the same that for the elliptic model, except for the number of load increments that has been increased to 103 (smaller initial load increments have been needed to obtain convergence of the boundary value problem). The distribution of

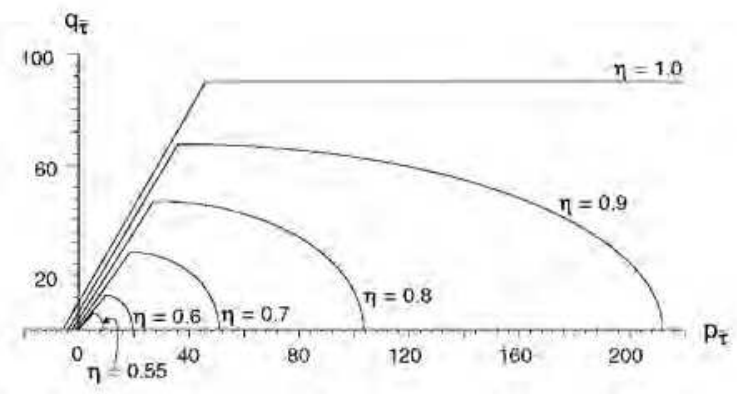

Figure 14. Traces of the cone-cap yield function on the meridian plane $q_{t}-p_{t}$ for different relative densities, $\eta$. Powder-A material parameters, see Table I. 

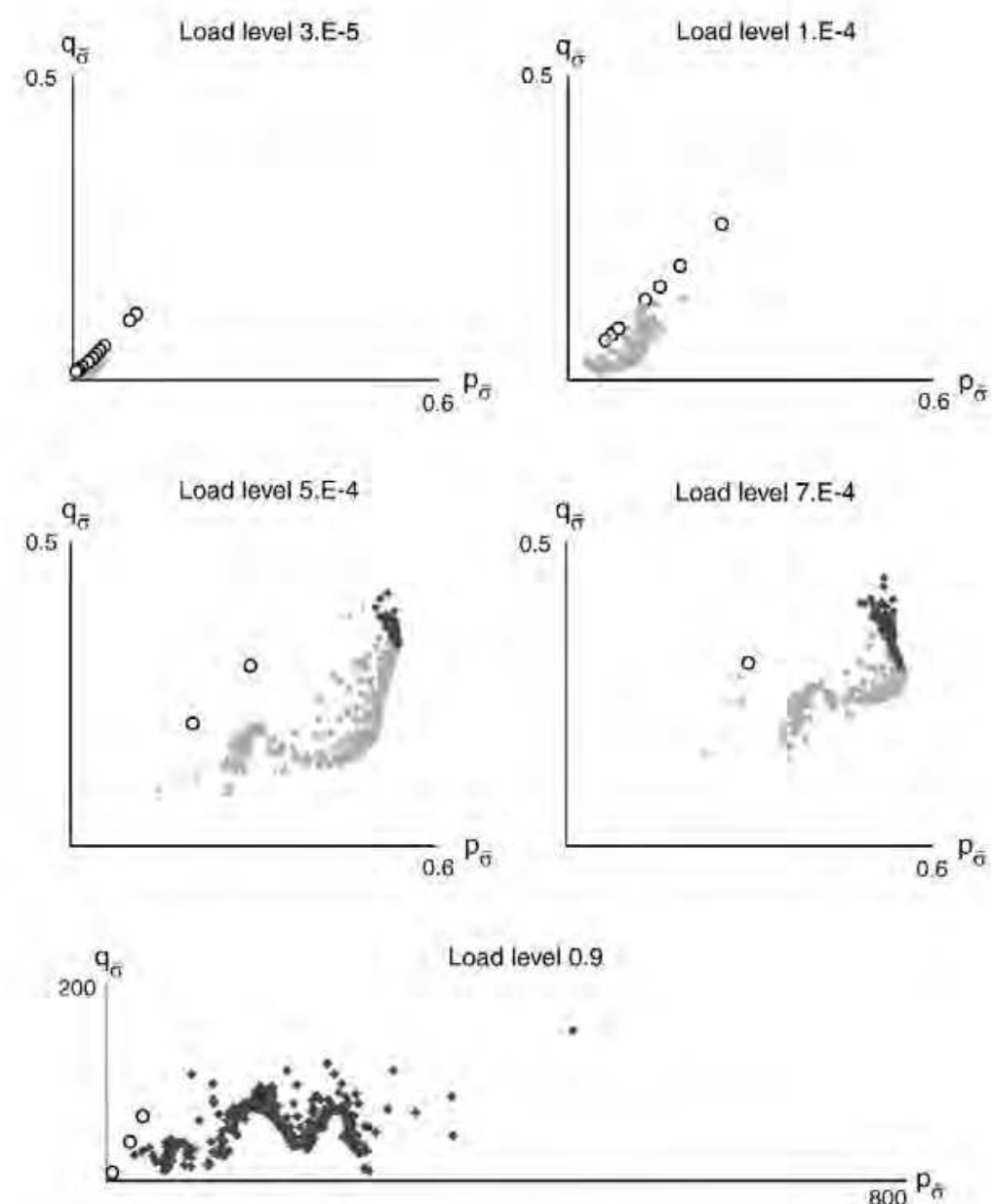

Load level 0.9

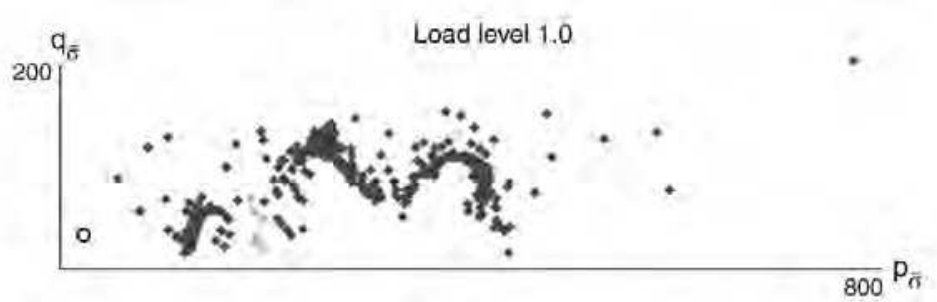

Figure 15. Frictionless compaction of the flanged component with the cone-cap model. Distribution of Cauchy stresses at Gauss-point level on the meridian plane $q_{\pi}-p_{\bar{\delta}}$ for different load levels. Plastic steps on the cap region (dark diamonds), plastic steps on the cone region (circles) and elastic steps (light diamonds).

Cauchy stresses at Gauss-point level on the meridian plane $q_{\sigma}-p_{\bar{\sigma}}$ for different load levels is depicted in Figure 15. Circles and dark marks correspond to points under plastic regime on the cone and cap regions respectively. Light marks denote elastic regime. The influence of the cone yield 
surface on the stress distribution is significant in the first steps of the test, but it reduces drastically to 1-3 Gauss points for the rest of test. The Gauss points with plastic loading in the cone region are those of the elements located on the left side of point $\mathrm{C}$. The distribution of Cauchy stresses at the end of the test (Figure 15, load level 1.0) is quite similar to that obtained with the elliptic model, Figure 13. The distribution of the relative density at the end of the test is shown in Figure 16. The results are also similar to those obtained with the elliptic model, Figure 11. The influence of the cone is restricted to a slight reduction of the relative density in the ABCG region. The evolution of the mean pressure of the top punch during the test with the elliptic model and the cone-cap model are depicted in Figure 17. These results agree with the previous ones: the influence of the cone is reduced. Moreover, it decreases as the compaction test progresses.

No direct comparison of powder compaction simulations with elliptic and cone-cap models has been found in the literature. The cone yield surface incorporates in the constitutive model the particle sliding that occurs at low pressures [29]. According to Cante et al. [24], this effect could be relevant at the transfer stage (the beginning of the industrial process, when an initial movement of the punches leads to the initial configuration of the sample). The present results show that, as expected, the effect of the cone yield surface is significant at the beginning of the compaction process, when a low pressure is applied. However, the influence is highly reduced as the compaction process advances, and the final results are quite similar with and without the cone (i.e. with the cone-cap model and with the elliptic model). The influence of the cone yield function when the friction effects between powder and compaction tools are important bas not been established yet.

The convergence results for different load levels are depicted in Figure 18. These load levels include those used in Figure 15 to show the distribution of Cauchy stresses at Gauss-point level. Quadratic convergence is found in all cases, for load increments with Gauss points under plastic loading in the cone region and with Gauss points under plastic loading in both regions.

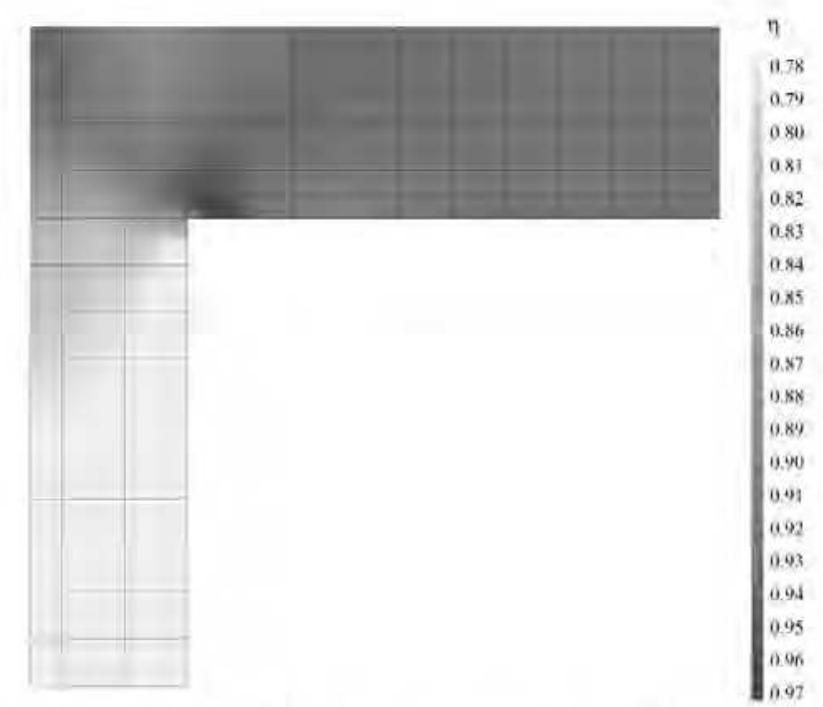

Figure 16. Frictionless compaction of the flanged component with the cone-cap model. Final relative density, $\eta$, distribution after a top displacement of $6.06 \mathrm{~mm}$ 


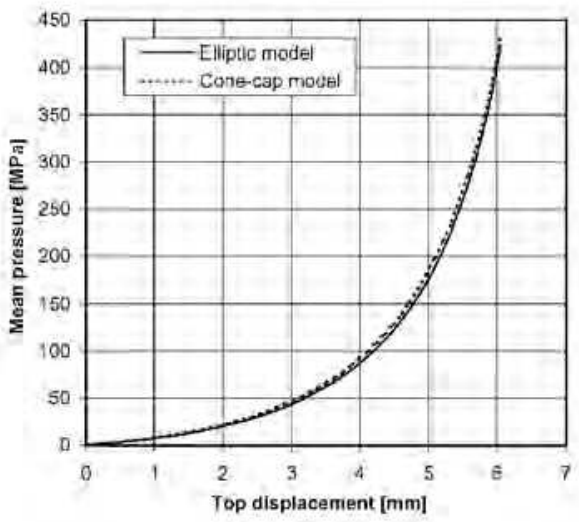

(a)

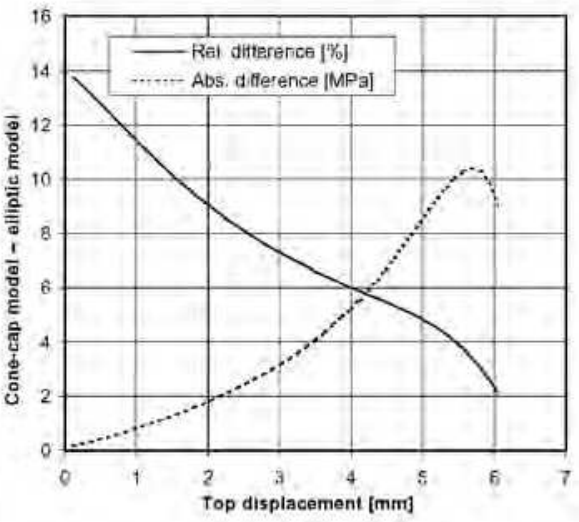

(b)

Figure 17. Frictionless compaction of the flanged component with the elliptic and the cone-cap models: (a) Mean pressure of the top punch versus top displacement; (b) relative and absolute difference of the two curves.
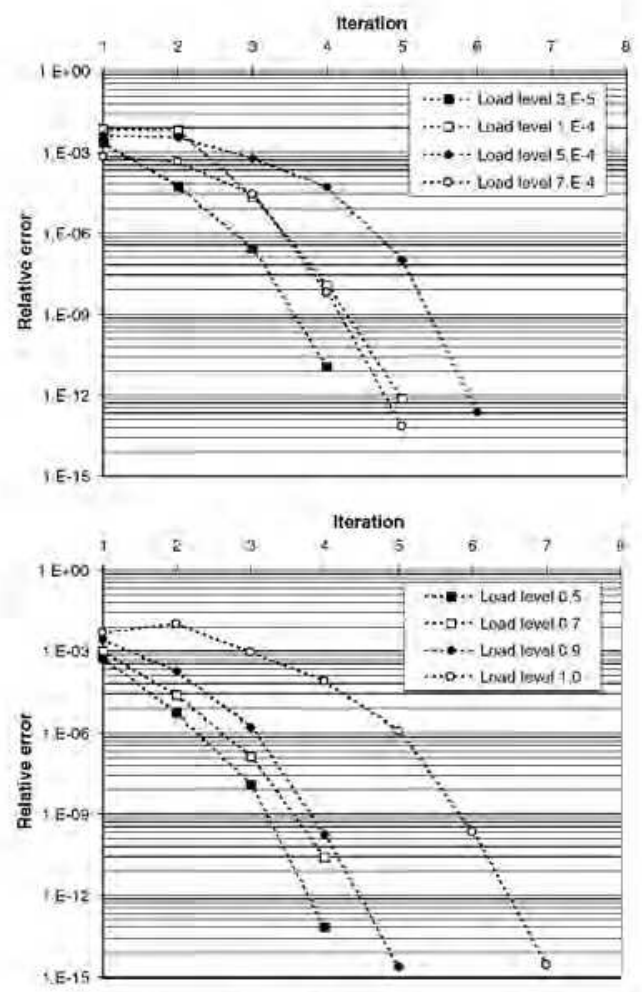

Figure 18. Frictionless compaction of the flanged component with the cone-cap model. Convergence results for different load levels. 


\section{CONCLUDING REMARKS}

The consistent tangent moduli for general isotropic multiplicative hyperelastic and densitydependent plastic models have been presented. Moreover, it has been shown that the inclusion of the density in the plastic equations does not modify the standard backward Euler return-mapping algorithm based on the exponential map [6,7].

Both elastic and plastic large deformations are included in the formulation. The yield function and flow rules are assumed to be expressed as general functions of the Kirchhoff stresses and the relative density. This includes the plastic models formulated in terms of Cauchy stresses $[20]$ as a particular case.

As in the density-independent case, the expression of the consistent tangent moduli is composed of two terms, the geometric part, which only depends on the trial state and the elastic energy function, and the material part, which depends on the plastic model. The influence of the density is restricted to the material part. At Gauss points under plastic loading, the material part is found to be composed of two terms. The standard one for density-independent plastic models $[6,9]$ and an additional one which includes the influence of the density on the plastic equations.

The computation of the additional term only requires some vector-matrix products and the derivatives of the plastic equations with respect to the relative density. Therefore, it does not represent any significant increase of the computational cost. On the other hand, the consistent tangent moduli are, in general, unsymmetric. This has to be taken into account when solving the linear system of equations in order to obtain quadratic convergence with the Newton-Raphson method.

Some representative examples with a Cauchy-based Drucker-Prager model, a density-dependent elliptic model and a density-dependent cone-cap model have been presented. In the examples involving the elliptic and the cone-cap models, the derivatives with respect to the relative density have been approximated numerically $[35,43]$. In all the examples quadratic convergence has been found.

The influence of neglecting the density part of the consistent tangent moduli has been analysed. It turns out that convergence is usually lost. When it is not, only linear convergence is obtained. The influence of symmetrizing the tangent is also assessed. Except for frictionless isostatic compaction tests (where the elliptic and cap model leads to symmetric tangents) linear convergence and, later, non-convergence are obtained.

A frictionless compaction test of an iron powder has also been simulated, as an example of a highly demanding boundary value problem from a computational point of view. An arbitrary Lagrangian-Eulerian formulation for multiplicative elastoplasticity [32] is used to avoid mesh distortion. The results obtained with the elliptic and the cone-cap models have been compared. Quadratic convergence has been found in both cases. Further analysis of compaction tests, including friction effects, are presented in Reference [25].

\section{APPENDIX A: DENSITY-DEPENDENT MATERIAL MODELS}

In the following, the three material models used in the examples, Section 4, are presented. Attention is focused in the expression of the yield functions. The corresponding flow vectors are directly related with them. Thus, only their definition and main features are analysed here. The complete expressions of the flow vectors can be derived in a straightforward manner. 
The yield functions presented below are expressed in terms of the inyariants

$$
I_{1}(\bar{\tau})=\sum_{i=1}^{n_{\mathrm{dim}}}[\bar{\tau}]_{i} \text { and } J_{2}(\bar{\tau})=\frac{1}{2} \sum_{i=1}^{n_{\mathrm{dim}}}\left([\operatorname{dev}(\bar{\tau})]_{i}\right)^{2}
$$

where $I_{1}(\bar{\tau})$ denotes the first invariant of $\bar{\tau}$ (recall that $\bar{\tau}$ is a vector whose components are the eigenvalues of the Kirchhoff stress tensor $\tau$ ) and $J_{2}(\bar{\tau})$ the second invariant of the deviatoric part of $\bar{\tau},\left\{[\operatorname{dev}(\bar{\tau})]_{i}=[\bar{\tau}]_{i}-I_{1}(\bar{\tau}) / 3\right\}_{i=1, \ldots, n_{\mathrm{dim}}}$. The following functions of $I_{1}(\bar{\tau})$ and $J_{2}(\bar{\tau})$ are also used

$$
p_{\bar{\tau}}=-\frac{I_{1}(\bar{\tau})}{3} \text { and } q_{\bar{\tau}}=\sqrt{3 I_{2}(\bar{\tau})}
$$

Remark A1. The invariants defined in Equations (A1) and (A2) are also applied to the vector $\overline{\boldsymbol{\sigma}}$, whose components are the eigenvalues of the Cauchy stress tensor $\sigma, \sigma=\sum_{i=1}^{n_{\text {dim }}}[\bar{\sigma}]_{i} \mathbf{n}_{\mathrm{tr}}^{i} \otimes \mathbf{n}_{t r}^{i}$. The relationship between $\bar{\tau}$ and $\bar{\sigma}$ follows from Equations (5) and (12).

$$
\overline{\boldsymbol{\sigma}}=\frac{\bar{\tau}}{\operatorname{det}(\boldsymbol{F})}=\frac{\eta}{\eta_{0}} \bar{\tau}
$$

Therefore, $I_{1}(\bar{\sigma}), J_{2}(\bar{\sigma}), p_{\bar{\sigma}}$ and $q_{\bar{\sigma}}$ are equal to the corresponding functions of $\bar{\tau}$ multiplied by $\eta / \eta_{0}$.

\section{A.1. Drucker-Prager model}

Two expressions of the Drucker-Prager model are considered: one with the yield function based on the principal Kirchhoff stresses (Kirchhoff-based Drucker-Prager model) and another based on the principal Cauchy stresses (Cauchy-based Drucker-Prager model). In both cases, the flow vector $\mathbf{m}_{\bar{\tau}}$ is set equal to the derivatives of the yield function with respect to the principal Kirchhoff stresses, $\mathbf{m}_{\bar{\tau}}=\partial f / \partial \bar{\tau}$.

The Kirchhoff-based Drucker-Prager (KDP) yield function is defined as

$$
f_{\mathrm{KDP}}(\bar{\tau})=\sqrt{2 J_{2}(\bar{\tau})}+\sqrt{\frac{2}{3}}\left(\tan \left(\phi_{\mathrm{DP}}\right) \frac{I_{1}(\bar{\tau})}{3}-C_{\mathrm{DP}}\right)
$$

with $\phi_{\mathrm{DP}}$ the friction angle and $C_{\mathrm{DP}}$ the cohesion. The intersection with the deviatoric axis $\left(p_{\bar{\tau}}=0\right)$ is at $q_{\bar{i}}=C_{\mathrm{DP}}$, and the cone vertex (the intersection with the hydrostatic axis, $q_{\bar{\tau}}=0$ ) is at $p_{\overline{\mathrm{t}}}=-C_{\mathrm{DP}} / \tan \left(\phi_{\mathrm{DP}}\right)$. The von Mises yield function is recovered for $\phi_{\mathrm{DP}}=0^{\circ}$.

The Cauchy-based Drucker-Prager (CDP) yield function, $f_{\mathrm{CDP}}(\bar{\sigma})$, is obtained after substitution of $\bar{\tau}$ by $\bar{\sigma}$ in Equation (44). In order to apply the developments presented in this paper, $f_{\mathrm{CDP}}(\overline{\boldsymbol{\sigma}})$ must be expressed in terms of the principal Kirchhoff stresses, $\bar{\tau}$, and the relative density, $\eta$. This can be done by substituting Equation (A3) in $f_{\mathrm{CDP}}(\bar{\sigma})$, see Remark 1, which leads to

$$
\tilde{f}_{\mathrm{CDP}}(\bar{\tau}, \eta)=\frac{\eta}{\eta_{0}} \sqrt{2 J_{2}(\bar{\tau})}+\sqrt{\frac{2}{3}}\left(\tan \left(\phi_{\mathrm{DP}}\right) \frac{\eta}{\eta_{0}} \frac{I_{1}(\bar{\tau})}{3}-C_{\mathrm{DP}}\right)
$$

However, $\tilde{f}_{\mathrm{CDP}}(\tilde{\tau}, \eta)$ is not used in the simulations of Section 4 . The equivalent yield function

$$
\tilde{\tilde{f}}_{\mathrm{CDP}}(\bar{\tau}, \eta)=\frac{\eta_{0}}{\eta} \tilde{f}_{\mathrm{CDP}}(\bar{\tau}, \eta)=\sqrt{2 J_{2}(\bar{\tau})}+\sqrt{\frac{2}{3}}\left(\tan \left(\phi_{\mathrm{DP}}\right) \frac{I_{1}(\bar{\tau})}{3}-\frac{\eta_{0}}{\eta} C_{\mathrm{DP}}\right)
$$


is preferred because it leads to a density-independent flow vector, $\partial \mathbf{m}_{\bar{i}} / \partial \eta=0$, contrary to Equation (A5), see Remark A2. The parameter $\eta_{0}$ is set equal to one for all $\boldsymbol{X}$, see Remark 1.

Remark A2. The yield functions given in Equations (A5) and (A6) are equivalent in the sense that, for a given trial state, both lead to the same solution on strains, stresses and internal variables of the plastic corrector problem, Equation (21). Therefore, both yield functions model in the same way the elastoplastic behaviour of the material. However, the plastic corrector problems are not the same because the respective incremental plastic multipliers, $\Delta \tilde{y}$ and $\Delta \tilde{\tilde{y}}$, are different. They are related through

$$
\Delta \tilde{\gamma}=\frac{\eta_{0}}{\eta} \Delta \tilde{\tilde{y}}
$$

On the other hand, the density-dependent part of the consistent tangent moduli of both problems is also different, see Equation (39). The yield function that leads to the simpler consistent tangent moduli, $\widetilde{\tilde{f}}_{\mathrm{CDP}}(\bar{\tau}, \eta)$, is used in this work.

\section{A.2. Elliptic model}

This model is defined by a density-dependent yield function (expressed in terms of the principal Kirchhoff stresses). The flow vector $\mathbf{m}_{\tilde{i}}$ is fixed equal to the derivatives of the yield function with respect to $\bar{\tau}$.

The yield function is [22]

$$
f_{\text {ellip }}(\bar{\tau}, \eta)=2 J_{2}(\bar{\tau})+a_{1}(\eta)\left(\frac{I_{1}(\bar{\tau})}{3}\right)^{2}-\frac{2}{3} a_{2}(\eta)\left(\sigma_{y}\right)^{2}
$$

with

$$
\begin{aligned}
& a_{1}(\eta)=\left\{\begin{array}{cc}
\left(\frac{1-\eta^{2}}{2+\eta^{2}}\right)^{n_{1}} & \eta<1 \\
0 & \eta \geqslant 1
\end{array}\right. \\
& a_{2}(\eta)= \begin{cases}\left(\frac{0.02 \eta_{0}}{1-0.98 \eta_{0}}\right)^{n_{2}} & \eta \leqslant \eta_{0} \\
\left(\frac{\eta-0.98 \eta_{0}}{1-0.98 \eta_{0}}\right)^{n_{2}} & \eta>\eta_{0}\end{cases}
\end{aligned}
$$

The value of $a_{1}(\eta)$ decreases from $2^{-n_{1}}$ at $\eta=0$ to zero for $\eta \geqslant 1$. The value of $a_{2}(\eta)$ increases monotonically from a minimum value for $\eta \leqslant \eta_{0}$ [21] and its value at $\eta=1$ is one. The intersection with the deviatoric axis is at $q_{\bar{\tau}}=\sqrt{a_{2}(\eta)} \sigma_{y}$ and the intersection with the hydrostatic axis is at $p_{\bar{\tau}}= \pm \sqrt{\left(2 a_{2}(\eta) / 3 a_{1}(\eta)\right)} \sigma_{y}$. Note that $f_{\text {ellip }}$ becomes the von Mises yield function for $\eta \geqslant 1$. 


\section{A.3. Cone-cap model}

This model is composed of two density-dependent yield functions: a cone defined by a Drucker-Prager yield function and a cap defined by an elliptic yield function. Both are expressed in terms of the principal Kirchhoff stresses. The flow vector is defined in two parts, one for the cone and the other for the cap. Both are presented in the following, after the yield function expressions.

The cone yield function is defined as

$$
f_{\text {cone }}(\bar{\tau}, \eta)=\sqrt{2 J_{2}(\bar{\tau})}+\sqrt{\frac{2}{3}}\left(\tan \left(\phi_{\text {cone }}(\eta)\right) \frac{I_{1}(\bar{\tau})}{3}-C_{\text {cone }}(\eta)\right)
$$

with

$$
\begin{gathered}
\phi_{\text {cone }}(\eta)= \begin{cases}\phi_{\min } & \eta \leqslant \eta_{0} \\
\left(\frac{\phi_{\min }-\phi_{\max }}{1+\eta_{0}^{2}-2 \eta_{0}}\right)\left(\eta^{2}-2 \eta+1\right)+\phi_{\text {max }} & 1 \leqslant \eta>\eta_{0} \\
\phi_{\text {max }} & \eta>1\end{cases} \\
C_{\text {cone }}(\eta)= \begin{cases}C_{\text {ref }}\left(\frac{0.02 \eta_{0}}{1-0.98 \eta_{0}}\right)^{n_{\circ}} & \eta \leqslant \eta_{0} \\
C_{\text {ref }}\left(\frac{\eta-0.98 \eta_{0}}{1-0.98 \eta_{0}}\right)^{n_{\circ}} & \eta>\eta_{0}\end{cases}
\end{gathered}
$$

The friction angle $\phi_{\text {cone }}(\eta)$ varies parabolically from a minimum value $\phi_{\min }$ at $\eta=\eta_{0}$ to a maximum value $\phi_{\max }$ at $\eta=1$. The cohesion $C_{\text {cone }}(\eta)$ increases as the function $a_{2}(\eta)$ of the elliptic model, Equation $(\mathrm{A} 9 \mathrm{~b})$. The reference value $C_{\mathrm{ref}}$ is reached at $\eta=1$.

The definition of the cap yield function is based on the elliptic yield function presented before, Equation (A8). It is defined in order to have the same maximum shear strength (maximum value of $q_{i}$ ) and the same hydrostatic compression strength (maximum value of $p_{\bar{\tau}}$ ), as the elliptic yield function, $\sqrt{a_{2}(\eta)} \sigma_{y}$ and $\sqrt{\left(2 a_{2}(\eta) / 3 a_{1}(\eta)\right)} \sigma_{y}$, respectively, Its expression is

$$
f_{\text {cap }}(\bar{\tau}, \eta)=2 J_{2}(\bar{\tau})+\tilde{a}_{1}(\eta)\left(\frac{I_{1}(\bar{\tau})-I_{\text {inter }}(\eta)}{3}\right)^{2}-\frac{2}{3} a_{2}(\eta)\left(\sigma_{y}\right)^{2}
$$

with

$$
\begin{aligned}
& I_{\text {inter }}(\eta)=3 \frac{C_{\text {cone }}(\eta)-\sqrt{a_{2}(\eta)} \sigma_{y}}{\tan \left(\phi_{\text {cone }}(\eta)\right)} \\
& I_{\text {compr }}(\eta)=-\sqrt{\frac{6 a_{2}(\eta)}{a_{1}(\eta)}} \sigma_{y} \\
& \tilde{a}_{1}(\eta)=\frac{6 a_{2}(\eta) \sigma_{y}^{2}}{\left(I_{\text {compr }}(\eta)-I_{\text {inter }}(\eta)\right)^{2}}
\end{aligned}
$$


and $a_{1}(\eta)$ and $a_{2}(\eta)$ given by Equations (A9). Note that the maximum shear strength is reached at cone-cap intersection, $I_{\text {inter }}(\eta)$, instead of $p_{\bar{\tau}}=0$ as the elliptic yield function does. Although Cante et al. [24] use $f_{\text {ellip }}$ defined in Equation (A8) as a cap yield function, in this work the expression of $f_{\text {cap }}$ given by Equation (A12) is preferred because it simplifies the flow vector definition, as shown in the following.

The flow vector $\mathbf{m}_{\bar{\tau}}$ is defined by parts, one expression for the cap region, $I_{1}(\bar{\tau}) \leqslant I_{\text {inter }}(\eta)$, and another for the cone region, $I_{1}(\bar{\tau})>I_{\text {inter }}(\eta)$. In the cap region, it is associative, so it is equal to the derivatives of the yield function with respect to $\bar{\tau}$,

$$
\mathbf{m}_{\bar{\tau} \text { cap }}=\frac{\partial f_{\text {cap }}}{\partial \bar{\tau}}
$$

In the cone region is non-associative, equal to

$$
\mathbf{m}_{\bar{\tau} \text { cone }}=\mathbf{A}(\eta) \frac{\partial f_{\text {cone }}}{\partial \bar{\tau}}
$$

with $\mathbf{A}(\eta)$ a matrix of order $n_{\mathrm{dim}}$ defined so that $\mathbf{m}_{\bar{\tau} \text { cone }}$ at cone-cap intersection is equal to $\mathbf{m}_{\bar{\tau}}$ (pure deviatoric) and at cone vertex is pure hydrostatic:

$$
\begin{aligned}
\mathbf{A}(\eta)= & \max \left\{1, \frac{3 J_{2}(\bar{\tau})}{a_{2}(\eta)\left(\sigma_{y}\right)^{2}}\right\}\left(\mathbf{I}_{n_{\mathrm{dio}}}-\frac{1}{3} \mathbf{1 1}^{\mathrm{T}}\right) \\
& +\max \left\{1, \frac{I_{\text {inter }}(\eta)-I_{1}(\bar{\tau})}{I_{\text {inter }}(\eta)+3 C_{\text {cone }}(\eta) \cot \left(\phi_{\text {cone }}(\eta)\right)}\right\}\left(\frac{1}{3} \mathbf{1 1}^{\mathrm{T}}\right)
\end{aligned}
$$

With the $\mathbf{m}_{\bar{\tau} \text { cone }}$ given by Equations (A15) and (A16) no corner return-mapping algorithm $[3,24, \mathrm{~A} 1, \mathrm{~A} 2]$ is needed in the cone-cap intersection and neither in the cone vertex. This reduces the computational effort of the numerical time-integration algorithm. The same approach has already been used to avoid the grey zone at cone-cap intersection in plastic models for granular materials $[40, \mathrm{~A} 4]$. A different technique, based on smoothing the yield function, has been used by Brandt and Nilsson [23] with the same purpose.

\section{REFERENCES}

1. Simo JC, Taylor RL. Consistent tangent operators for rate-independent elastoplasticity. Computer Methods in Apptied Mechanics and Engineering 1985; 48:101-118.

2. Runesson K, Samuelsson A, Bernspang L. Numerical technique in plasticity including solution advancement control. International Journal for Numerical Methods in Engineering 1986; 22:769-788.

3. Simo JC, Kennedy JG, Govindjee S. Non-smooth multisurface plasticity and viscoplasticity. Loading/unloading conditions and numerical al gorithms. International Journal for Numerical Methods in Engineering 1988;26:2161-2185.

4. Crisfield MA. Non-Linear Finite Element Analysis of Solids and Structures. 2 Advanced Topics. Wiley: Chichester, 1997.

5. Pérez-Foguet A, Rodríguez-Ferran A, Huerta A. Consistent tangent matrices for substepping schemes. Computer Methods in Applied Mechanics and Engineering, 2001, to appear.

6. Simo JC. Algorithms for static and dynamic multiplicative plasticity that preserve the classical return mapping schemes of the infinitesimal theory. Computer Methods in Apptied Mechanics and Engineering 1992; 99:61-112.

7. Cuitiño A, Ortiz M. A material-independent method for extending stress update algorithms from small-strain plasticity to finite plasticity with multiplicative kinematics. Engineering Computations 1992; 9:437-451.

8. Perić D, Owen DRJ, Honnor MEA. A model for finite strain elasto-plasticity based on logarithmic strains: computational issues. Computer Methods in Applied Mechanics and Engineering 1992; 94:35-61. 
9. Simo JC. Numerical analysis and simulation of plasticity. In Handbook of Numerical Analysis, Ciarlet PG, Lions JL (eds), vol. VI. Elsevier: Amsterdam, 1998; 179-499.

10. Simo JC, Miehe C. Associative coupled thermoplasticity at finite strains: formulation, numerical analysis and implementation. Computer Methods in Applied Mechanics and Engineering 1992; 98:41-104.

11. de Souza Neto EA, Perić D, Owen DRJ. A model for elastoplastic damage at finite strains: algorithmic issues and applications. Engineering Computations 1994; 11:257-281.

12. Li X. Large strain constitutive modelling and computation for isotropic, creep elastoplastic damage solids. International Journal for Numerical Methods in Engineering 1995; 38:841-860.

13. Simo JC, Meschke G. A new class of algorithms for classical plasticity extended to finite strains. Application to geomaterials. Computational Mechanics 1993; 11:253-278.

14. Meschke G, Liu G, Mang HA. Large strain finite-element analysis of snow. Journal of Engineering Mechanics 1996; 122(7):591-602.

15. Borja RI, Tamagnini C. Cam-clay plasticity. Part III: extension of the infinitesimal model to include finite strains. Computer Methods in Applied Mechanics and Engineering 1998; 155:73-95.

16. Callari C, Auricchio F, Sacco E. A finite-strain Cam-Clay model in the framework of multiplicative elasto-plasticity International Journal of Plasticity 1998; 14(12):1155-1187.

17. Borja RI, Alarcón E. A mathematical framework for finite strain elastoplastic consolidation. Part I: balance laws, variational formulation, and linearization. Computer Methods in Applied Mechanics and Engineering 1995; 122: $145-171$

18. Borja RI, Alarcón E. Elastoplastic consolidation at finite strain. Part II: finite element implementation and numerical examples. Computer Methods in Applied Mechanics and Engineering 1998; 159:103-122.

19. Armero F. Formulation and finite element implementation of a multiplicative model of couple poro-plasticity at finite strains under fully saturated conditions. Computer Methods in Applied Mechanics and Engineering 1999; 171:205-241.

20. Meschke G, Liu WN. A re-formulation of the exponential algorithm for finite strain plasticity in terms of cauchy stresses. Computer Methods in Applied Mechanics and Engineering 1999; 173:167-187.

21. Oliver J, Oller S, Cante JC. Numerical simulation of uniaxial compaction processes in powder materials. Proceedings of the International Congress on Numerical Methods in Engineering and Applied Sciences, Chile, 1992; 1277-1286.

22. Oliver J, Oller S, Cante JC. A plasticity model for simulation of industrial powder compaction processes. International Journal of Solids and Structures 1996; 33(20-22):3161-3178.

23. Brandt J, Nilsson L. A constitutive model for compaction of granular media, with account for deformation induced anisotropy. Mechanics of Cohesive-Frictional Materials 1999; 4(4):391-418.

24. Cante JC, Oliver J, Hernández R. Simulación numérica de las etapas de transferencia y prensado del proceso de compactación de pulvimateriales. In Abascal R, Domínguez J, Bugeda G (eds.), IV Congreso de Métodos Numéricos en Ingeniería, SEMNI, Spain, 1999.

25. Perez-Foguet A, Rodríguez-Ferran A, Huerta A. Application of an ALE formulation for density-dependent hyperelastic-plalstic models to the simulation of powder compaction processes. In Habraken AM. (Ed.), 4th International ESAFORM Conference on Material Forming, Liege, Belgium, 2001.

26. Jinka AGK, Bellet M, Fourment L. A new three-dimensional finite element model for the simulation of powder forging processes: application to hot forming of P/M connecting rod. International Journal for Numerical Methods in Engineering 1997; 40:3955-3978.

27. Ragab AR, Saleh ChAR. Evaluation of constitutive models for voided solids. International Journal of Plasticity 1999 15:1041-1065

28. Aydin I, Briscoe BJ, Sanliturk KY. The internal form of compacted ceramic components. A comparison of a finite-element modelling with experiment. Powder Technology 1996; 89(3):239-254.

29. Lewis RW, Khoei AR. Numerical modelling of large deformation in metal powder forming. Computer Methods in Applied Mechanics and Engineering 1998; 159:291-328.

30. Brandt J, Nilsson L. FE-simulation of compaction and solid-state sintering of cemented carbides. Mechanics of Cohesive-Frictional Materials 1998; 3(2):181-205.

31. Khoei AR, Lewis RW. Adaptive finite element remeshing in a large deformation analysis of metal powder forming International Journal for Numerical Methods in Engineering 1999; 45:801-820.

32. Rodríguez-Ferran A, Pérez-Foguet A, Huerta A. Arbitrary Lagrangina-Eulerian (ALE) formulation for hyperelastoplasticity. International Journal for Numerical Methods in Engineering, 2000, in press.

33. Bonet J, Wood RD. Nonlinear Continum Mechanics for Finite Element Analysis. Cambridge University Press: Cambridge, 1997.

34. Ortiz M, Martin JB. Symmetry-preserving return mapping algorithms and incrementally extremal paths: a unification of concepts. International Journal for Numerical Methods in Engineering 1989; 28:1839-1853.

35. Pérez-Foguet A, Rodríguez-Ferran A, Huerta A. Numerical differentiation for local and global tangent operators in computational plasticity. Computer Methods in Applied Mechanics and Engineering 2000; 189:277-296.

36. Dorémus P, Geindreau C, Martin A, Lécot R, Dao M. High pressure triaxial apparatus for investigating the mechanical behaviour of metal powders. Proceedings of Powder Metallurgy 94, World Congress, Paris, 1994; $733-736$. 


\section{CONSISTENT TANGENT MATRICES}

37. Ernst E, Barnekow D. Pressure, friction and density during axial powder compaction. Proceedings of Powder Metalhurgy 94, World Congress, Paris, 1994; 673-676

38. Shinohara K, Oida M, Golman B. Effect of particle shape on angle of internal friction by triaxial compression test. Powder Technology 2000; 107:131-136.

39. Doremus P, Toussaint F. Simple tests standard procedure for the characterisation of green compacted powder. Institut National Polytechnique de Grenoble, Laboratoire Sols Solides Structures, 2000.

40. P'erez-Foguet A, Huerta A. Plastic flow potential for the cone region of the MRS-Lade model. Journal of Engineering Mechanics 1999; 125(3):364-367.

41. Pramono E, Willam K. Implicit integration of composite yield surfaces with corners. Engineering Computations 1989; 6:186-197.

42. Hofstetter G, Simo JC, Taylor RL. A modified cap model: closest point solution algorithms. Computers and Structures. 1993; 46(2):203-214

43. Pérez-Foguet A, Rodríguez-Ferran A, Huerta A. Numerical differentiation for non-trivial consistent tangent matrices: an application to the MRS-Lade model. International Journal for Numerical Methods in Engineering 2000; 48:159-184.

44. Macari EJ, Weihe S, Arduino P. Implicit integration of elastoplastic constitutive models for frictional materials with highly non-linear hardening functions. Mechanics of Cohesive-Frictionat Materials 1997; 2:1-29. 